\title{
NALPS: a precisely dated European climate record 120-60 ka
}

\author{
R. Boch ${ }^{1,2}$, H. Cheng ${ }^{2,3}$, C. Spötl ${ }^{1}$, R. L. Edwards ${ }^{2}$, X. Wang ${ }^{2,4}$, and Ph. Häuselmann ${ }^{5}$ \\ ${ }^{1}$ Institut für Geologie und Paläontologie, Universität Innsbruck, Austria \\ ${ }^{2}$ Department of Geology and Geophysics, University of Minnesota, Minneapolis, USA \\ ${ }^{3}$ Institute of Global Environmental Change, Xi' an Jiaotong University, Xi' an, China \\ ${ }^{4}$ Lamont-Doherty Earth Observatory of Columbia University, Palisades, NY, USA \\ ${ }^{5}$ Swiss Institute of Speleology and Karst Sciences - SISKA, La Chaux-de-Fonds, Switzerland \\ Received: 7 March 2011 - Published in Clim. Past Discuss.: 30 March 2011 \\ Revised: 12 October 2011 - Accepted: 12 October 2011 - Published: 24 November 2011
}

\begin{abstract}
Accurate and precise chronologies are essential in understanding the rapid and recurrent climate variations of the Last Glacial - known as Dansgaard-Oeschger (D-O) events - found in the Greenland ice cores and other climate archives. The existing chronological uncertainties during the Last Glacial, however, are still large. Radiometric age data and stable isotopic signals from speleothems are promising to improve the absolute chronology. We present a record of several precisely dated stalagmites from caves located at the northern rim of the Alps (NALPS), a region that favours comparison with the climate in Greenland. The record covers most of the interval from 120 to $60 \mathrm{ka}$ at an average temporal resolution of 2 to $22 \mathrm{yr}$ and $2 \sigma$-age uncertainties of ca. 200 to $500 \mathrm{yr}$. The rapid and large oxygen isotope shifts of 1 to $4.5 \%$ occurred within decades to centuries and strongly mimic the Greenland D-O pattern. Compared to the updated Greenland ice-core timescale (GICC05modelext) the NALPS record confirms the timing of rapid warming and cooling transitions between 118 and $106 \mathrm{ka}$, but suggests younger ages for D-O events between 106 and $60 \mathrm{ka}$. As an exception, the timing of the rapid transitions into and out of the stadial following GI 22 is earlier in NALPS than in the Greenland ice-core timescale. In addition, there is a discrepancy in the duration of this stadial between the icecore and the stalagmite chronology (ca. 2900 vs. $3650 \mathrm{yr}$ ). The short-lived D-O events 18 and 18.1 are not recorded in NALPS, provoking questions with regard to the nature and
\end{abstract}

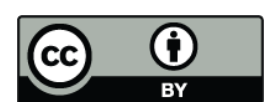

Correspondence to: R. Boch (ronny.boch@uibk.ac.at) the regional expression of these events. NALPS resolves recurrent short-lived climate changes within the cold Greenland stadial and warm interstadial successions, i.e. abrupt warming events preceding GI 21 and 23 (precursor-type events) and at the end of GI 21 and 25 (rebound-type events), as well as intermittent cooling events during GI 22 and 24. Such superimposed events have not yet been documented outside Greenland.

\section{Introduction}

In the Greenland ice cores drastic climate changes are documented during the Last Glacial period. The rapid and recurrent variations - known as Dansgaard-Oeschger (D-O) events (Dansgaard et al., 1993; Grootes et al., 1993) - are expressed as relatively warm and humid Greenland Interstadials (GI) and relatively cold and dry Greenland Stadials (GS; see Rousseau et al., 2006; Lowe et al., 2008 for an event-stratigraphical recommendation). These successions had a global effect on climate (e.g. Clement and Peterson, 2008). Large and rapid air temperature changes occurred within a few years to a few decades (Steffensen et al., 2008). The amplitude was largest in the N-Atlantic realm and reached $8-16^{\circ} \mathrm{C}$ during rapid warmings in Greenland (Severinghaus et al., 1998; Lang et al., 1999; Huber et al., 2006; Capron et al., 2010). The millennial-scale changes also affected the concentration of greenhouse gases (Grachev et al., 2007; Loulergue et al., 2008), as well as global sea-level and high-latitude ice-sheets (Lambeck and Chappell, 2001; Arz et al., 2007). Recently, the occurrence of three types

Published by Copernicus Publications on behalf of the European Geosciences Union. 
of short-lived, sub-millennial climate events was discussed (Capron et al., 2010). These events occurred within D-O cycles and consist of abrupt warmings either preceding individual GI (named precursor events) or occurring toward the end of some of the GI (named rebound events), as well as of abrupt coolings, e.g. during GI 24 (Capron et al., 2010).

The D-O variations show a quasi-periodic occurrence of ca. 1470 yr (Bond et al., 1997; Grootes and Stuiver, 1997; Rahmstorf, 2003), although a recent study suggested that the recurrence interval is not robust and thus not significant (Peavoy and Franzke, 2010). Different triggers and mechanisms have been invoked to explain these rapid climate changes. Among these are freshwater influx into the North Atlantic (Clark et al., 2001; Arz et al., 2007), solar variations (e.g. Braun et al., 2005), internal oscillations (Broecker et al., 1990; Birchfield et al., 1994; Rahmstorf, 2002) and stochastic resonance (Alley et al., 2001; Claussen et al., 2003; Ditlevsen and Johnsen, 2010). Moreover, changes and different states of the Atlantic Meridional Overturning Circulation (AMOC) are discussed as internal forcings (Ahn and Brook, 2008; Schmittner and Galbraith, 2008), including latitudinal shifts in the location of North Atlantic Deep Water production (e.g. Ganopolski and Rahmstorf, 2002). Others claim a tropical trigger of the D-O variability (Clement and Cane, 1999; Clement and Peterson, 2008), or a rapidly changing windfield due to the dynamics of continental ice sheets (Wunsch, 2006). Ditlevsen and Johnsen (2010) recently reported evidence that internal noise triggered $\mathrm{D}-\mathrm{O}$ warmings and concluded that these events cannot be predicted.

Accurate and precise chronologies are fundamental to improve our understanding of the enigmatic D-O pattern and its global teleconnections. The existing chronological uncertainties during the Last Glacial, however, are still significant (Svensson et al., 2008) and various timescales are available for Greenland ice cores alone (Meese et al., 1997; Johnsen et al., 2001; Svensson et al., 2008; Wolff et al., 2010). The multi-parameter, annual layer-counted GICC05 timescale (Svensson et al., 2008) covers the past $60 \mathrm{kyr}$ and age uncertainties are on the order of $2.5 \mathrm{kyr}$ at $60 \mathrm{ka}$. For the first half of the Last Glacial (ca. 118-60 ka) the GICC05modelext timescale (Wolff et al., 2010) was recently suggested as an improvement of the previous ss09sea timescale (North Greenland Ice Core Project members, 2004). A comparison of the ice-core chronologies with those from other Last Glacial archives, e.g. N-Atlantic deep-marine sediments, suffers from the radiocarbon dating limit and uncertainties associated with the ${ }^{14} \mathrm{C}$-calibration and the marine reservoir effect. U-Th-dated speleothem chronologies are promising and help to reduce the dating uncertainties substantially. Previous studies showed that speleothems capture the D-O pattern and provide valuable contributions (e.g. Wang et al., 2001, 2007, 2008; Spötl et al., 2006; Genty et al., 2003; Cruz et al., 2005; Dykoski et al., 2005; Drysdale et al., 2007; Cheng et al., 2009; Fleitmann et al., 2009; Asmerom et al., 2010).
In the Alps, which are known to be a climatically sensitive region (Casty et al., 2005; Auer et al., 2007), the speleothem $\mathrm{O}$ isotopic composition constitutes a climate proxy that allows for a direct comparison with the Greenland $\mathrm{O}$ isotope records (e.g. von Grafenstein et al., 1999; Spötl and Mangini, 2002). In particular, the Alps and Greenland share a dominant Atlantic influence and the common $\mathrm{O}$ isotopic signal allows comparison of the chronology of the ice-cores to that of radiometrically dated speleothems.

In this study we present a record consisting of several precisely dated stalagmites from caves located at the northern rim of the European Alps (NALPS). This region is exposed to a strong Atlantic influence via northwesterly winds thus favouring a comparison with climate in Greenland. Moreover, the cave host rock favours deposition of speleothems with excellent geochemical dating characteristics. All Last Glacial samples exhibit sharp $\mathrm{O}$ isotope transitions highly reminiscent of the D-O pattern seen in the ice cores. Our record covers most of the first half of the Last Glacial period (118-64 ka) at high temporal resolution.

\section{Cave sites and stalagmite samples}

Four cave sites were selected for this study (Fig. 1). Beatus Cave is located in central Switzerland and samples were collected in a gallery at $875 \mathrm{~m}$ a.s.l. The cave air temperature is ca. $8{ }^{\circ} \mathrm{C}$ and mean annual precipitation is $1258 \mathrm{~mm}$ (20042008; meteorological station Interlaken, ca. $8 \mathrm{~km}$ from the cave). Baschg Cave is located in the westernmost part of Austria and the cave entrance is at $780 \mathrm{~m}$ a.s.l. The cave air temperature is ca. $10^{\circ} \mathrm{C}$ and mean annual precipitation is $1231 \mathrm{~mm}$ (1971-2000; meteorological station Feldkirch, ca. $5 \mathrm{~km}$ from the cave). Klaus-Cramer and Schneckenloch Caves are located close to each other on the margin of the high-alpine Gottesacker karst plateau in western Austria (Fig. 1). Klaus-Cramer Cave is a shallow cave and the entrance opens at $1964 \mathrm{~m}$ a.s.l. The cave air temperature is only ca. $1-2{ }^{\circ} \mathrm{C}$. The entrance of Schneckenloch Cave is at ca. $1270 \mathrm{~m}$ and the cave air temperature is ca. $6.5^{\circ} \mathrm{C}$. Mean annual precipitation at both sites is $1908 \mathrm{~mm}$ (19712000; station Schoppernau, $835 \mathrm{~m}$ a.s.l. and ca. $7 \mathrm{~km}$ from the caves). All four caves have small and well-defined catchments (a few $\mathrm{km}^{2}$ only) and developed in the same carbonate host rock (Lower Cretaceous Schrattenkalk Formation). This host rock provides favourable geochemical conditions for U-Th dating, i.e. high U (0.5-2 ppm) and low detrital Th concentrations (typically $0.2-6 \mathrm{ppb}^{232} \mathrm{Th}$ ). Therefore, no significant correction of the U-Th ages is needed and the resulting ages are precise and accurate. Two stalagmites were recovered from Beatus, three from Baschg, one from Klaus-Cramer, and one from Schneckenloch Cave. Some samples were found broken while others were still in growth position. The stalagmites are typically small in size, i.e. between 11 and $38 \mathrm{~cm}$ high and near-equal in diameter 


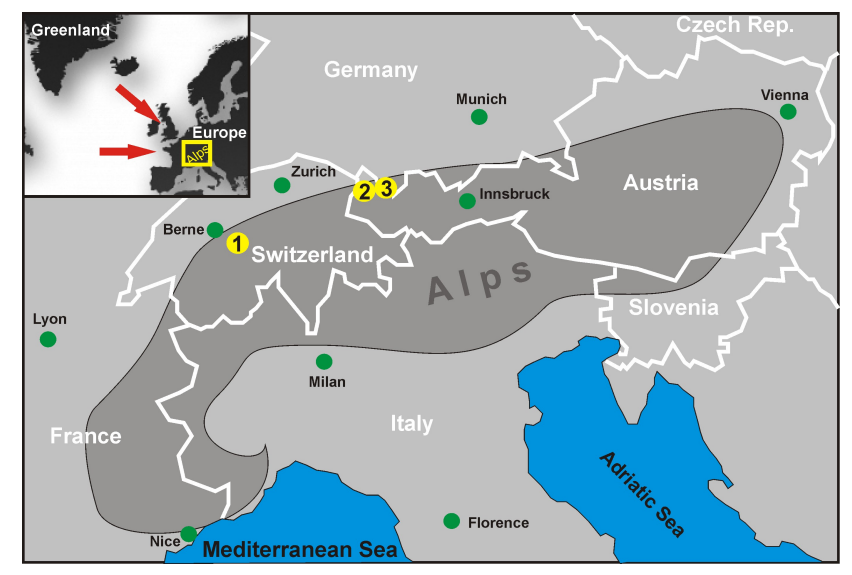

Fig. 1. Location of the selected cave sites at the northern rim of the Alps: (1) Beatus Cave (Switzerland); (2) Baschg Cave (Austria); (3) Klaus-Cramer- and Schneckenloch Caves (Austria). The northern rim of the Alps is dominated by moisture advection from the Atlantic Ocean (red arrows).

along their growth axes. The samples consist of dense calcite and some show distinct lamination. Minor portions of some samples (typically near the base) consist of impure calcite due to clay and organic inclusions. These sections were not used in the combined record. Interestingly, speleothem growth locally also occurred during cold stadial conditions. This places tight constraints on the minimum temperatures of these alpine caves.

\section{Methods}

The stalagmites were cut in half and a 0.5 to $1 \mathrm{~cm}$-thick slab was cut from the axial part and polished. Subsamples for radiometric U-Th dating (typically 0.05 to $0.1 \mathrm{~g}$ ) were obtained using a dentist drill. After dissolving the powders in nitric acid and adding a mixed ${ }^{229} \mathrm{Th}^{233} \mathrm{U}^{236} \mathrm{U}$ spike, $\mathrm{U}$ and Th were separated from each other and from matrix elements using co-precipitation with $\mathrm{Fe}$ and an ion-exchange resin in Teflon columns (procedure similar to Edwards et al., 1986). The isotopic compositions of $\mathrm{U}$ and $\mathrm{Th}$ of the majority of the samples were analysed using a ThermoFinnigan NEPTUNE multi-collector inductively-coupled plasma mass spectrometer (MC-ICP-MS; Cheng et al., 2009). The remaining samples were analysed on a ThermoFinnigan ELEMENT singlecollector ICP-MS (Shen et al., 2008). In both cases a spiked NBL-112A standard solution was measured before and after the sample runs and blank measurements were conducted to correct for the $\mathrm{U}$ and $\mathrm{Th}$ backgrounds. Isotopic activity ratios were calculated using the new decay constants of Cheng et al. (2008). The final ages were corrected for detrital Th using an initial ${ }^{230} \mathrm{Th} /{ }^{232} \mathrm{Th}$ activity ratio of 0.8 (cf. Richards and Dorale, 2003). Ages are quoted in "a BP" (years before 1950 A.D.; see Table 1). The U-Th-based age models (cf. Fig. S1 in the Supplement) were calculated using the open-source $\mathrm{R}$ statistics software (version 2.10.0; R Development Core Team, 2010) and an algorithm optimised for speleothems (Scholz and Hoffmann, 2011). The age-depth function and the corresponding $95 \%$-confidence intervals were calculated by superposition of ensembles of piecewise linear fits. In addition to the U-Th data points and corresponding errors the algorithm also uses stratigraphic information, i.e. the age of the speleothem must increase with increasing distance from top.

Stalagmite slabs and thin sections were investigated using transmitted-light, epifluorescence, as well as reflectedlight microscopy. Subsamples for stable oxygen and carbon isotopic analysis were micromilled at 0.15 to $0.25 \mathrm{~mm}$ resolution along the central stalagmite growth axes. The isotopic compositions were analysed using a ThermoFisher Deltaplus XL isotope ratio mass spectrometer coupled to a ThermoFisher GasBench II. Results are reported relative to the VPDB standard and the precision of the $\delta^{18} \mathrm{O}$ and $\delta^{13} \mathrm{C}$ values is 0.08 and $0.06 \%$ (1-sigma), respectively (Spötl and Vennemann, 2003). In this paper, we focus on the palaeoclimatic application of the $\mathrm{O}$ isotopic signal.

\section{The NALPS stalagmite record}

The new $\mathrm{O}$ isotope record from the northern Alps covers the time interval from ca. 120 to $60 \mathrm{ka}$, i.e. D-O 25 to D-O 18 (data are available in the Supplement). Interstadials dominate the record, reflecting favourable climate conditions with regard to speleothem growth. Speleothem formation, however, continued at least during some of the stadials, indicating that the caves were not frozen during these times. The record from seven stalagmites is temporally constrained by 154 U-Th ages (20-30 per stalagmite; Table 1$)$. Typical relative $2 \sigma$-uncertainties range from $0.2-0.6 \%$, i.e. average uncertainties associated with the timing and duration of rapid climate changes range from ca. 200 to $500 \mathrm{yr}$ (stalagmites KC1 $210 \mathrm{yr}$, BA1-clean section $410 \mathrm{yr}, \mathrm{BA} 1 \mathrm{~b} 350 \mathrm{yr}$, BA2 340 yr, EXC3 450 yr, EXC4 400 yr, SCH7 530 yr; cf. Fig. 2).

The NALPS stable isotope curves consist of ca. 8200 individual analyses and show rapid and large isotope shifts of up to $4.5 \%$ underscoring the high sensitivity of these cave sites. The average temporal resolution ranges from 2 to $22 \mathrm{yr}$ depending on the stalagmite and time interval. With regard to the transitions, a sharp (rapid) central portion is often flanked by more gradual progressions towards the isotopic maxima and minima. This could either be an expression of the regional climate, reflect hydrological processes in the karst aquifer, or could in part be a smoothing artefact of the applied age model. Regarding the climatic interpretation of the alpine speleothem $\mathrm{O}$ isotopic signal, the pattern is highly reminiscent of Greenland, i.e. high $\delta^{18} \mathrm{O}$ values represent warm interstadials and low values cold stadials (Fig. 2). The speleothem $\mathrm{O}$ isotope values primarily 
Table 1. U-Th dating results of seven stalagmites from four cave sites.

\begin{tabular}{|c|c|c|c|c|c|c|c|c|c|c|}
\hline Sample & $\begin{array}{l}{ }^{238} \mathrm{U} \\
{[\mathrm{ppb}]}\end{array}$ & $\begin{array}{c}{ }^{232} \mathrm{Th} \\
{[\mathrm{ppt}]}\end{array}$ & $\begin{array}{c}{ }^{230} \mathrm{Th} /{ }^{232} \mathrm{Th} \\
{\left[\text { atomic } \times 10^{-6} \text { ] }\right.}\end{array}$ & $\begin{array}{c}\delta^{234} \mathrm{U}^{\mathrm{a}} \\
\text { [measured] }\end{array}$ & $\begin{array}{c}{ }^{230} \mathrm{Th} /{ }^{238} \mathrm{U} \\
\text { [activity] }\end{array}$ & $\begin{array}{c}\text { Age [a] } \\
\text { [uncorr.] }\end{array}$ & $\begin{array}{c}\text { Age [a] } \\
\text { [corr.] }\end{array}$ & $\begin{array}{c}\delta^{234} \mathrm{U}_{\text {Initial }}^{\mathrm{b}} \\
\text { [corr.] }\end{array}$ & $\begin{array}{l}\text { Age [a BP] }]^{\mathrm{c}} \\
{[\text { corr.] }}\end{array}$ & $\begin{array}{c}\text { DFT }[\mathrm{mm}]^{\mathrm{d}} \\
\text { isotrack-scale }\end{array}$ \\
\hline KC1-0.1 & $224.5 \pm 0.3$ & $3920 \pm 80$ & $830 \pm 20$ & $1142.0 \pm 3.0$ & $0.8800 \pm 0.0020$ & $54840 \pm 190$ & $54620 \pm 250$ & $1332.0 \pm 3.0$ & $54560 \pm 250$ & 1.0 \\
\hline $\mathrm{KC} 1-0.2$ & $294.1 \pm 0.3$ & $1310 \pm 30$ & $3320 \pm 70$ & $1084.0 \pm 2.0$ & $0.8980 \pm 0.0020$ & $58240 \pm 150$ & $58180 \pm 160$ & $1277.0 \pm 3.0$ & $58120 \pm 160$ & 2.3 \\
\hline KC1-0.5 & $334.0 \pm 1.0$ & $580 \pm 10$ & $8660 \pm 120$ & $1090.0 \pm 8.0$ & $0.9130 \pm 0.0050$ & $59290 \pm 480$ & $59260 \pm 480$ & $1289.0 \pm 9.0$ & $59200 \pm 480$ & 4.5 \\
\hline KC1-0.8 & $415.2 \pm 0.3$ & $640 \pm 10$ & $9840 \pm 200$ & $1013.0 \pm 2.0$ & $0.9220 \pm 0.0010$ & $63100 \pm 140$ & $63080 \pm 140$ & $1211.0 \pm 3.0$ & $63020 \pm 140$ & 7.5 \\
\hline KC1-1.0 & $463.0 \pm 1.0$ & $470 \pm 10$ & $14830 \pm 300$ & $990.0 \pm 2.0$ & $0.9190 \pm 0.0020$ & $63830 \pm 160$ & $63820 \pm 160$ & $1185.0 \pm 2.0$ & $63760 \pm 160$ & 9.8 \\
\hline $\mathrm{KC} 1-2.0$ & $536.0 \pm 1.0$ & $142 \pm 3$ & $57360 \pm 1300$ & $984.0 \pm 2.0$ & $0.9220 \pm 0.0020$ & $64300 \pm 160$ & $64300 \pm 160$ & $1179.0 \pm 2.0$ & $64240 \pm 160$ & 19.5 \\
\hline $\mathrm{KC} 1-3.0$ & $607.0 \pm 1.0$ & $420 \pm 10$ & $21850 \pm 450$ & $981.0 \pm 2.0$ & $0.9260 \pm 0.0020$ & $64750 \pm 170$ & $64750 \pm 170$ & $1178.0 \pm 3.0$ & $64690 \pm 170$ & 30.0 \\
\hline $\mathrm{KC} 1-4.5$ & $485.0 \pm 0.4$ & $1040 \pm 20$ & $7240 \pm 150$ & $971.0 \pm 2.0$ & $0.9380 \pm 0.0010$ & $66350 \pm 150$ & $66330 \pm 150$ & $1170.0 \pm 3.0$ & $66270 \pm 150$ & 45.0 \\
\hline KC1-6.0 & $565.0 \pm 1.0$ & $240 \pm 10$ & $36610 \pm 760$ & $972.0 \pm 2.0$ & $0.9510 \pm 0.0020$ & $67550 \pm 190$ & $67540 \pm 190$ & $1176.0 \pm 3.0$ & $67480 \pm 190$ & 60.0 \\
\hline KC1-7.9 & $620.0 \pm 1.0$ & $550 \pm 10$ & $17870 \pm 360$ & $958.0 \pm 2.0$ & $0.9570 \pm 0.0020$ & $68760 \pm 170$ & $68750 \pm 170$ & $1163.0 \pm 2.0$ & $68690 \pm 170$ & 79.2 \\
\hline KC1-8.9 & $644.0 \pm 1.0$ & $330 \pm 10$ & $31690 \pm 650$ & $975.0 \pm 2.0$ & $0.9750 \pm 0.0020$ & $69640 \pm 180$ & $69630 \pm 180$ & $1187.0 \pm 3.0$ & $69570 \pm 180$ & 88.5 \\
\hline KC1-9.6 & $768.0 \pm 1.0$ & $210 \pm 4$ & $59000 \pm 1230$ & $964.0 \pm 2.0$ & $0.9770 \pm 0.0020$ & $70330 \pm 220$ & $70330 \pm 220$ & $1176.0 \pm 3.0$ & $70270 \pm 220$ & 96.5 \\
\hline KC1-10.1 & $815.0 \pm 1.0$ & $1650 \pm 30$ & $8040 \pm 160$ & $975.0 \pm 2.0$ & $0.9860 \pm 0.0020$ & $70590 \pm 180$ & $70570 \pm 180$ & $1190.0 \pm 3.0$ & $70510 \pm 180$ & 100.5 \\
\hline KC1-10.6 & $1011.0 \pm 1.0$ & $151 \pm 3$ & $107570 \pm 2440$ & $953.0 \pm 2.0$ & $0.9750 \pm 0.0020$ & $70690 \pm 200$ & $70690 \pm 200$ & $1163.0 \pm 3.0$ & $70630 \pm 200$ & 106.0 \\
\hline KC1-11.0 & $910.0 \pm 1.0$ & $138 \pm 3$ & $106290 \pm 2420$ & $949.0 \pm 2.0$ & $0.9750 \pm 0.0020$ & $70900 \pm 200$ & $70900 \pm 200$ & $1159.0 \pm 3.0$ & $70840 \pm 200$ & 110.0 \\
\hline $\mathrm{KC} 1-11.3$ & $1166.0 \pm 2.0$ & $280 \pm 10$ & $66270 \pm 1390$ & $954.0 \pm 2.0$ & $0.9770 \pm 0.0020$ & $70880 \pm 200$ & $70880 \pm 200$ & $1165.0 \pm 3.0$ & $70820 \pm 200$ & 113.0 \\
\hline KC1-11.7 & $925.0 \pm 1.0$ & $710 \pm 10$ & $21090 \pm 430$ & $952.0 \pm 2.0$ & $0.9780 \pm 0.0020$ & $71070 \pm 180$ & $71060 \pm 180$ & $1164.0 \pm 3.0$ & $71000 \pm 180$ & 116.6 \\
\hline KC1-12.0 & $1133.0 \pm 2.0$ & $143 \pm 3$ & $128810 \pm 2770$ & $955.0 \pm 2.0$ & $0.9840 \pm 0.0020$ & $71470 \pm 240$ & $71470 \pm 240$ & $1169.0 \pm 3.0$ & $71410 \pm 240$ & 120.0 \\
\hline KC1-12.8 & $1180.0 \pm 3.0$ & $340 \pm 10$ & $56310 \pm 1270$ & $951.0 \pm 3.0$ & $0.9850 \pm 0.0040$ & $71760 \pm 390$ & $71760 \pm 390$ & $1165.0 \pm 4.0$ & $71700 \pm 390$ & 128.0 \\
\hline KC1-13.1 & $916.0 \pm 1.0$ & $710 \pm 10$ & $21160 \pm 430$ & $974.0 \pm 2.0$ & $0.9960 \pm 0.0020$ & $71720 \pm 210$ & $71710 \pm 210$ & $1192.0 \pm 3.0$ & $71650 \pm 210$ & 131.1 \\
\hline $\mathrm{KC} 1-13.6$ & $1148.0 \pm 1.0$ & $450 \pm 10$ & $41300 \pm 850$ & $954.0 \pm 2.0$ & $0.9890 \pm 0.0020$ & $72010 \pm 190$ & $72000 \pm 190$ & $1169.0 \pm 3.0$ & $71940 \pm 190$ & 136.0 \\
\hline BA1b-0.2 & $395.1 \pm 0.4$ & $250 \pm 10$ & $17730 \pm 370$ & $340.0 \pm 2.0$ & $0.6870 \pm 0.0010$ & $75590 \pm 230$ & $75580 \pm 230$ & $421.0 \pm 2.0$ & $75520 \pm 230$ & 2.4 \\
\hline BA1b-1.0 & $287.0 \pm 1.0$ & $480 \pm 10$ & $6960 \pm 100$ & $369.0 \pm 3.0$ & $0.7060 \pm 0.0030$ & $75960 \pm 560$ & $75930 \pm 560$ & $457.0 \pm 4.0$ & $75870 \pm 560$ & 10.0 \\
\hline BA1b-1.4 & $445.0 \pm 1.0$ & $410 \pm 10$ & $12680 \pm 260$ & $360.0 \pm 2.0$ & $0.7010 \pm 0.0010$ & $76010 \pm 230$ & $75990 \pm 230$ & $446.0 \pm 2.0$ & $75930 \pm 230$ & 14.0 \\
\hline BA $1 b-1.7$ & $457.0 \pm 1.0$ & $350 \pm 10$ & $14840 \pm 300$ & $357.0 \pm 2.0$ & $0.6980 \pm 0.0010$ & $75810 \pm 250$ & $75790 \pm 250$ & $442.0 \pm 2.0$ & $75730 \pm 250$ & 17.0 \\
\hline BA1b-1.9 & $416.0 \pm 1.0$ & $350 \pm 10$ & $13570 \pm 280$ & $358.0 \pm 2.0$ & $0.7010 \pm 0.0010$ & $76200 \pm 230$ & $76190 \pm 230$ & $443.0 \pm 2.0$ & $76130 \pm 230$ & 19.4 \\
\hline BA1b-2.3 & $338.1 \pm 0.3$ & $11910 \pm 240$ & $310 \pm 10$ & $282.0 \pm 2.0$ & $0.6710 \pm 0.0010$ & $78210 \pm 220$ & $77450 \pm 580$ & $351.0 \pm 2.0$ & $77390 \pm 580$ & 23.0 \\
\hline BA1b-2.4 & $496.0 \pm 1.0$ & $230 \pm 10$ & $25460 \pm 540$ & $357.0 \pm 2.0$ & $0.7100 \pm 0.0010$ & $77690 \pm 240$ & $77680 \pm 240$ & $444.0 \pm 2.0$ & $77620 \pm 240$ & 24.0 \\
\hline BA $1 \mathrm{~b}-3.0$ & $613.0 \pm 1.0$ & $187 \pm 4$ & $38250 \pm 810$ & $348.0 \pm 2.0$ & $0.7080 \pm 0.0010$ & $78170 \pm 260$ & $78160 \pm 260$ & $434.0 \pm 2.0$ & $78100 \pm 260$ & 30.4 \\
\hline BA1b-4.0 & $659.0 \pm 1.0$ & $2340 \pm 50$ & $3350 \pm 70$ & $367.0 \pm 2.0$ & $0.7200 \pm 0.0010$ & $78370 \pm 230$ & $78300 \pm 240$ & $457.0 \pm 2.0$ & $78240 \pm 240$ & 40.0 \\
\hline BA $1 b-5.8$ & $691.0 \pm 1.0$ & $450 \pm 10$ & $18080 \pm 370$ & $362.0 \pm 2.0$ & $0.7190 \pm 0.0010$ & $78650 \pm 230$ & $78630 \pm 230$ & $452.0 \pm 2.0$ & $78570 \pm 230$ & 58.0 \\
\hline BA1b-6.8 & $490.0 \pm 1.0$ & $1630 \pm 30$ & $3570 \pm 70$ & $355.0 \pm 2.0$ & $0.7180 \pm 0.0010$ & $79120 \pm 230$ & $79050 \pm 230$ & $444.0 \pm 2.0$ & $78990 \pm 230$ & 68.0 \\
\hline BA1b- 8.5 & $519.0 \pm 1.0$ & $260 \pm 10$ & $23460 \pm 490$ & $359.0 \pm 2.0$ & $0.7210 \pm 0.0010$ & $79180 \pm 240$ & $79170 \pm 240$ & $448.0 \pm 2.0$ & $79110 \pm 240$ & 85.0 \\
\hline BA1b-8.9 & $523.0 \pm 1.0$ & $2050 \pm 30$ & $3010 \pm 60$ & $345.0 \pm 2.0$ & $0.7160 \pm 0.0010$ & $79640 \pm 220$ & $79560 \pm 220$ & $432.0 \pm 2.0$ & $79500 \pm 220$ & 89.4 \\
\hline BA1b-9.5 & $516.0 \pm 1.0$ & $2870 \pm 60$ & $2150 \pm 40$ & $358.0 \pm 2.0$ & $0.7270 \pm 0.0010$ & $80170 \pm 240$ & $80060 \pm 260$ & $448.0 \pm 2.0$ & $80000 \pm 260$ & 95.0 \\
\hline BA1b-11.0 & $507.0 \pm 1.0$ & $880 \pm 20$ & $6770 \pm 140$ & $336.0 \pm 2.0$ & $0.7140 \pm 0.0010$ & $80120 \pm 270$ & $80090 \pm 270$ & $422.0 \pm 2.0$ & $80030 \pm 270$ & 109.8 \\
\hline BA1b-12.0 & $422.0 \pm 1.0$ & $310 \pm 10$ & $16000 \pm 390$ & $336.0 \pm 4.0$ & $0.7120 \pm 0.0030$ & $79910 \pm 620$ & $79900 \pm 620$ & $421.0 \pm 5.0$ & $79840 \pm 620$ & 119.8 \\
\hline BA1b-12.9 & $435.0 \pm 1.0$ & $20100 \pm 400$ & $260 \pm 10$ & $336.0 \pm 2.0$ & $0.7190 \pm 0.0020$ & $80900 \pm 290$ & $79960 \pm 730$ & $421.0 \pm 2.0$ & $79900 \pm 730$ & 128.8 \\
\hline BA1b-13.7 & $561.0 \pm 1.0$ & $1160 \pm 20$ & $5800 \pm 120$ & $350.0 \pm 2.0$ & $0.7260 \pm 0.0010$ & $80700 \pm 260$ & $80660 \pm 260$ & $440.0 \pm 2.0$ & $80600 \pm 260$ & 137.0 \\
\hline BA1b-14.5 & $333.3 \pm 0.3$ & $17940 \pm 360$ & $223 \pm 4$ & $344.0 \pm 2.0$ & $0.7280 \pm 0.0010$ & $81680 \pm 250$ & $80580 \pm 810$ & $432.0 \pm 2.0$ & $80520 \pm 810$ & 145.0 \\
\hline BA1-0.5 & $785.0 \pm 1.0$ & $5270 \pm 110$ & $1770 \pm 40$ & $334.0 \pm 2.0$ & $0.7190 \pm 0.0010$ & $81210 \pm 260$ & $81070 \pm 280$ & $419.0 \pm 2.0$ & $81010 \pm 280$ & 5 \\
\hline BA1-0.9 & $706.0 \pm 2.0$ & $1860 \pm 10$ & $4510 \pm 30$ & $334.0 \pm 3.0$ & $0.7190 \pm 0.0030$ & $81050 \pm 530$ & $81000 \pm 530$ & $420.0 \pm 3.0$ & $80940 \pm 530$ & 10 \\
\hline BA1-3.0 & $732.0 \pm 1.0$ & $1060 \pm 20$ & $8180 \pm 170$ & $327.0 \pm 2.0$ & $0.7160 \pm 0.0010$ & $81410 \pm 290$ & $81380 \pm 290$ & $411.0 \pm 2.0$ & $81320 \pm 290$ & 30 \\
\hline BA1-5.5 & $669.0 \pm 1.0$ & $620 \pm 10$ & $12880 \pm 170$ & $345.0 \pm 2.0$ & $0.7290 \pm 0.0030$ & $81670 \pm 530$ & $81660 \pm 530$ & $435.0 \pm 3.0$ & $81600 \pm 530$ & 56 \\
\hline BA1-9.5 & $825.0 \pm 1.0$ & $5170 \pm 100$ & $1930 \pm 40$ & $351.0 \pm 2.0$ & $0.7340 \pm 0.0010$ & $81880 \pm 250$ & $81750 \pm 270$ & $443.0 \pm 2.0$ & $81690 \pm 270$ & 95 \\
\hline BA1-10.0 & $539.0 \pm 1.0$ & $9480 \pm 190$ & $690 \pm 10$ & $351.0 \pm 2.0$ & $0.7350 \pm 0.0020$ & $82160 \pm 300$ & $81810 \pm 390$ & $442.0 \pm 2.0$ & $81750 \pm 390$ & 100 \\
\hline BA1-10.6 & $829.0 \pm 1.0$ & $3860 \pm 80$ & $2610 \pm 50$ & $351.0 \pm 2.0$ & $0.7370 \pm 0.0010$ & $82530 \pm 270$ & $82430 \pm 270$ & $443.0 \pm 2.0$ & $82370 \pm 270$ & 106 \\
\hline BA1-13.9 & $643.0 \pm 1.0$ & $3360 \pm 70$ & $2300 \pm 50$ & $332.0 \pm 2.0$ & $0.7270 \pm 0.0010$ & $82700 \pm 270$ & $82590 \pm 280$ & $419.0 \pm 2.0$ & $82530 \pm 280$ & 139 \\
\hline BA1-16.2 & $459.0 \pm 1.0$ & $4810 \pm 20$ & $1140 \pm 10$ & $325.0 \pm 3.0$ & $0.7260 \pm 0.0030$ & $83160 \pm 630$ & $82940 \pm 630$ & $411.0 \pm 4.0$ & $82880 \pm 630$ & 159 \\
\hline BA1-16.6 & $454.0 \pm 1.0$ & $10280 \pm 210$ & $520 \pm 10$ & $308.0 \pm 2.0$ & $0.7190 \pm 0.0010$ & $83600 \pm 290$ & $83130 \pm 440$ & $389.0 \pm 2.0$ & $83070 \pm 440$ & 166 \\
\hline BA1-17.4 & $443.8 \pm 0.4$ & $26460 \pm 510$ & $202 \pm 4$ & $303.0 \pm 1.0$ & $0.7320 \pm 0.0010$ & $86420 \pm 240$ & $85160 \pm 920$ & $385.0 \pm 2.0$ & $85100 \pm 920$ & 174 \\
\hline BA1-17.9 & $573.0 \pm 1.0$ & $6090 \pm 120$ & $1130 \pm 20$ & $305.0 \pm 2.0$ & $0.7290 \pm 0.0010$ & $85640 \pm 280$ & $85410 \pm 320$ & $388.0 \pm 2.0$ & $85350 \pm 320$ & 179 \\
\hline BA $1-18.3$ & $545.0 \pm 1.0$ & $3600 \pm 70$ & $1810 \pm 40$ & $304.0 \pm 2.0$ & $0.7260 \pm 0.0010$ & $85220 \pm 280$ & $85090 \pm 290$ & $387.0 \pm 2.0$ & $85030 \pm 290$ & 181 \\
\hline BA1-19.0 & $381.4 \pm 0.4$ & $6990 \pm 140$ & $660 \pm 10$ & $313.0 \pm 2.0$ & $0.7350 \pm 0.0010$ & $85840 \pm 260$ & $85460 \pm 370$ & $399.0 \pm 2.0$ & $85400 \pm 370$ & 190 \\
\hline BA1-20.8 & $467.0 \pm 1.0$ & $2290 \pm 50$ & $2440 \pm 50$ & $290.0 \pm 2.0$ & $0.7260 \pm 0.0010$ & $86670 \pm 290$ & $86560 \pm 300$ & $370.0 \pm 2.0$ & $86500 \pm 300$ & 207 \\
\hline BA1-22.0 & $549.0 \pm 1.0$ & $22180 \pm 440$ & $290 \pm 10$ & $249.0 \pm 1.0$ & $0.7120 \pm 0.0010$ & $88840 \pm 280$ & $87950 \pm 690$ & $319.0 \pm 2.0$ & $87890 \pm 690$ & 220 \\
\hline BA1-22.5 & $624.0 \pm 1.0$ & $2060 \pm 40$ & $3530 \pm 70$ & $244.0 \pm 2.0$ & $0.7080 \pm 0.0010$ & $88650 \pm 290$ & $88580 \pm 290$ & $314.0 \pm 2.0$ & $88520 \pm 290$ & 225 \\
\hline BA1-23.6 & $614.0 \pm 1.0$ & $3610 \pm 70$ & $1990 \pm 40$ & $243.0 \pm 1.0$ & $0.7080 \pm 0.0010$ & $88760 \pm 270$ & $88630 \pm 290$ & $312.0 \pm 2.0$ & $88570 \pm 290$ & 236 \\
\hline BA $1-24.2$ & $829.0 \pm 1.0$ & $3860 \pm 80$ & $168 \pm 3$ & $239.0 \pm 2.0$ & $0.7160 \pm 0.0010$ & $90730 \pm 330$ & $89160 \pm 1160$ & $307.0 \pm 3.0$ & $89100 \pm 1160$ & 242 \\
\hline BA1-24.9 & $319.0 \pm 1.0$ & $29560 \pm 130$ & $128 \pm 1$ & $230.0 \pm 4.0$ & $0.7180 \pm 0.0060$ & $92250 \pm 1300$ & $90150 \pm 1650$ & $296.0 \pm 5.0$ & $90090 \pm 1650$ & 246 \\
\hline BA1-25.2 & $469.0 \pm 1.0$ & $53270 \pm 1070$ & $109 \pm 2$ & $280.0 \pm 2.0$ & $0.7510 \pm 0.0020$ & $92360 \pm 420$ & $89910 \pm 1790$ & $361.0 \pm 3.0$ & $89850 \pm 1790$ & 252 \\
\hline BA1-25.9 & $580.0 \pm 1.0$ & $39630 \pm 790$ & $178 \pm 4$ & $267.0 \pm 2.0$ & $0.7380 \pm 0.0010$ & $91510 \pm 320$ & $90020 \pm 1100$ & $344.0 \pm 3.0$ & $89960 \pm 1100$ & 259 \\
\hline BA1-26.4 & $746.0 \pm 1.0$ & $56260 \pm 1130$ & $160 \pm 3$ & $266.0 \pm 2.0$ & $0.7340 \pm 0.0010$ & $90870 \pm 300$ & $89230 \pm 1200$ & $342.0 \pm 2.0$ & $89170 \pm 1200$ & 264 \\
\hline BA2-0.2 & $635.0 \pm 1.0$ & $490 \pm 10$ & $15060 \pm 310$ & $240.0 \pm 2.0$ & $0.7050 \pm 0.0010$ & $88590 \pm 310$ & $88570 \pm 310$ & $308.0 \pm 2.0$ & $88510 \pm 310$ & 1.8 \\
\hline BA2-0.5 & $486.0 \pm 1.0$ & $7220 \pm 150$ & $790 \pm 20$ & $240.0 \pm 2.0$ & $0.7100 \pm 0.0010$ & $89370 \pm 260$ & $89040 \pm 350$ & $309.0 \pm 2.0$ & $88980 \pm 350$ & 4.6 \\
\hline BA2-0.7 & $655.0 \pm 1.0$ & $1170 \pm 20$ & $6530 \pm 130$ & $240.0 \pm 2.0$ & $0.7050 \pm 0.0010$ & $88590 \pm 370$ & $88550 \pm 370$ & $308.0 \pm 3.0$ & $88490 \pm 370$ & 7 \\
\hline BA2-1.1 & $474.0 \pm 1.0$ & $650 \pm 10$ & $8400 \pm 170$ & $233.0 \pm 2.0$ & $0.7040 \pm 0.0010$ & $89090 \pm 290$ & $89050 \pm 290$ & $300.0 \pm 2.0$ & $88990 \pm 290$ & 11 \\
\hline BA2-1.6 & $517.0 \pm 1.0$ & $1140 \pm 20$ & $5320 \pm 110$ & $242.0 \pm 2.0$ & $0.7090 \pm 0.0010$ & $89030 \pm 330$ & $88980 \pm 340$ & $311.0 \pm 2.0$ & $88920 \pm 340$ & 16.0 \\
\hline BA2-2.0 & $616.0 \pm 1.0$ & $2390 \pm 50$ & $3050 \pm 60$ & $256.0 \pm 2.0$ & $0.7180 \pm 0.0010$ & $89060 \pm 290$ & $88970 \pm 300$ & $329.0 \pm 2.0$ & $88910 \pm 300$ & 20.0 \\
\hline BA2-2.4 & $904.0 \pm 1.0$ & $2210 \pm 40$ & $4880 \pm 100$ & $260.0 \pm 2.0$ & $0.7220 \pm 0.0010$ & $89300 \pm 320$ & $89250 \pm 320$ & $335.0 \pm 2.0$ & $89190 \pm 320$ & 24.4 \\
\hline BA2-3.0 & $919.0 \pm 1.0$ & $6070 \pm 120$ & $1790 \pm 40$ & $258.0 \pm 2.0$ & $0.7190 \pm 0.0020$ & $89040 \pm 340$ & $88900 \pm 360$ & $332.0 \pm 2.0$ & $88840 \pm 360$ & 30.0 \\
\hline BA2-3.3 & $807.0 \pm 1.0$ & $1580 \pm 30$ & $6070 \pm 120$ & $258.0 \pm 2.0$ & $0.7220 \pm 0.0010$ & $89500 \pm 320$ & $89450 \pm 320$ & $333.0 \pm 2.0$ & $89390 \pm 320$ & 33.0 \\
\hline BA2-3.7 & $372.5 \pm 0.3$ & $2220 \pm 40$ & $2000 \pm 40$ & $258.0 \pm 2.0$ & $0.7220 \pm 0.0010$ & $89490 \pm 270$ & $89360 \pm 280$ & $332.0 \pm 2.0$ & $89300 \pm 280$ & 37.4 \\
\hline BA2-4.5 & $434.0 \pm 1.0$ & $7070 \pm 140$ & $730 \pm 20$ & $254.0 \pm 2.0$ & $0.7230 \pm 0.0010$ & $90230 \pm 310$ & $89880 \pm 400$ & $328.0 \pm 2.0$ & $89820 \pm 400$ & 45.0 \\
\hline BA2-5.5 & $408.4 \pm 0.4$ & $1240 \pm 30$ & $3910 \pm 80$ & $258.0 \pm 2.0$ & $0.7210 \pm 0.0010$ & $89480 \pm 290$ & $89410 \pm 290$ & $332.0 \pm 2.0$ & $89350 \pm 290$ & 55.0 \\
\hline BA2-5.9 & $449.0 \pm 1.0$ & $700 \pm 10$ & $7590 \pm 150$ & $257.0 \pm 2.0$ & $0.7220 \pm 0.0010$ & $89590 \pm 320$ & $89560 \pm 330$ & $331.0 \pm 2.0$ & $89500 \pm 330$ & 58.6 \\
\hline BA2-6.3 & $444.1 \pm 0.4$ & $3170 \pm 60$ & $1670 \pm 30$ & $259.0 \pm 2.0$ & $0.7230 \pm 0.0010$ & $89550 \pm 310$ & $89390 \pm 330$ & $334.0 \pm 2.0$ & $89330 \pm 330$ & 63.0 \\
\hline BA2-6.9 & $477.0 \pm 1.0$ & $970 \pm 20$ & $5860 \pm 120$ & $258.0 \pm 2.0$ & $0.7250 \pm 0.0010$ & $90150 \pm 300$ & $90100 \pm 300$ & $333.0 \pm 2.0$ & $90040 \pm 300$ & 69.0 \\
\hline BA2-7.1 & $528.0 \pm 1.0$ & $1050 \pm 20$ & $5940 \pm 120$ & $253.0 \pm 2.0$ & $0.7200 \pm 0.0010$ & $89730 \pm 320$ & $89680 \pm 320$ & $326.0 \pm 2.0$ & $89620 \pm 320$ & 70.8 \\
\hline BA2-7.8 & $431.0 \pm 1.0$ & $3540 \pm 70$ & $1450 \pm 30$ & $253.0 \pm 2.0$ & $0.7210 \pm 0.0010$ & $89960 \pm 330$ & $89780 \pm 350$ & $326.0 \pm 2.0$ & $89720 \pm 350$ & 78.0 \\
\hline BA2-8.4 & $483.0 \pm 1.0$ & $7840 \pm 160$ & $730 \pm 20$ & $247.0 \pm 2.0$ & $0.7170 \pm 0.0010$ & $90050 \pm 300$ & $89690 \pm 390$ & $318.0 \pm 2.0$ & $89630 \pm 390$ & 83.6 \\
\hline BA2-8.5 & $380.9 \pm 0.4$ & $2990 \pm 60$ & $1500 \pm 30$ & $248.0 \pm 2.0$ & $0.7160 \pm 0.0010$ & $89620 \pm 280$ & $89450 \pm 310$ & $319.0 \pm 2.0$ & $89390 \pm 310$ & 85.0 \\
\hline BA2-9.1 & $339.6 \pm 0.3$ & $9230 \pm 190$ & $440 \pm 10$ & $265.0 \pm 2.0$ & $0.7290 \pm 0.0010$ & $90030 \pm 280$ & $89440 \pm 500$ & $341.0 \pm 2.0$ & $89380 \pm 500$ & 91.4 \\
\hline
\end{tabular}


Table 1. Continued.

\begin{tabular}{|c|c|c|c|c|c|c|c|c|c|c|}
\hline Sample & $\begin{array}{l}{ }^{238} \mathrm{U} \\
{[\mathrm{ppb}]}\end{array}$ & $\begin{array}{c}232 \mathrm{Th} \\
{[\mathrm{ppt}]}\end{array}$ & $\begin{array}{c}{ }^{230} \mathrm{Th} /{ }^{232} \mathrm{Th} \\
{\left[\text { atomic } \times 10^{-6} \text { ] }\right.}\end{array}$ & $\begin{array}{c}\delta^{234} \mathrm{U}^{\mathrm{a}} \\
\text { [measured] }\end{array}$ & $\begin{array}{c}{ }^{230} \mathrm{Th} /{ }^{238} \mathrm{U} \\
\text { [activity] }\end{array}$ & $\begin{array}{l}\text { Age [a] } \\
\text { [uncorr.] }\end{array}$ & $\begin{array}{c}\text { Age [a] } \\
\text { [corr.] }\end{array}$ & $\begin{array}{c}\delta^{234} \mathrm{U}_{\text {Initial }}^{\mathrm{b}} \\
\text { [corr.] }\end{array}$ & $\begin{array}{l}\text { Age [a BP] } \\
\text { [corr.] }\end{array}$ & $\begin{array}{l}\text { DFT }[\mathrm{mm}] \quad \mathrm{d} \\
\text { isotrack-scale }\end{array}$ \\
\hline EXC3-0.2 & $1070.0 \pm 1.0$ & $240 \pm 10$ & $51710 \pm 1090$ & $148.0 \pm 2.0$ & $0.7050 \pm 0.0010$ & $101000 \pm 400$ & $101000 \pm 400$ & $197.0 \pm 2.0$ & $100940 \pm 400$ & 2.0 \\
\hline EXC3-1.7 & $1554.0 \pm 2.0$ & $125 \pm 3$ & $144100 \pm 3300$ & $143.0 \pm 2.0$ & $0.7020 \pm 0.0010$ & $101060 \pm 350$ & $101060 \pm 350$ & $191.0 \pm 2.0$ & $101000 \pm 350$ & 17.0 \\
\hline EXC3-2.9 & $1709.0 \pm 3.0$ & $400 \pm 10$ & $49380 \pm 1010$ & $144.0 \pm 2.0$ & $0.7040 \pm 0.0010$ & $101370 \pm 420$ & $101360 \pm 420$ & $192.0 \pm 2.0$ & $101300 \pm 420$ & 29.4 \\
\hline EXC3-4.1 & $1450.0 \pm 3.0$ & $87 \pm 2$ & $193690 \pm 4760$ & $146.0 \pm 2.0$ & $0.7080 \pm 0.0020$ & $101900 \pm 470$ & $101900 \pm 470$ & $195.0 \pm 2.0$ & $101840 \pm 470$ & 40.7 \\
\hline EXC3-4.2 & $1403.0 \pm 2.0$ & $174 \pm 4$ & $93640 \pm 2040$ & $148.0 \pm 2.0$ & $0.7060 \pm 0.0010$ & $101230 \pm 390$ & $101230 \pm 390$ & $197.0 \pm 2.0$ & $101170 \pm 390$ & 41.4 \\
\hline EXC3-4.3 & $1071.0 \pm 1.0$ & $500 \pm 10$ & $25090 \pm 510$ & $149.0 \pm 1.0$ & $0.7070 \pm 0.0010$ & $101250 \pm 300$ & $101240 \pm 300$ & $199.0 \pm 2.0$ & $101180 \pm 300$ & 42.9 \\
\hline EXC3-4.4 & $1254.0 \pm 2.0$ & $470 \pm 10$ & $31460 \pm 640$ & $151.0 \pm 1.0$ & $0.7150 \pm 0.0010$ & $102680 \pm 380$ & $102670 \pm 380$ & $202.0 \pm 2.0$ & $102610 \pm 380$ & 44.4 \\
\hline EXC3-4.7 & $970.0 \pm 3.0$ & $30 \pm 10$ & $437650 \pm 110310$ & $153.0 \pm 3.0$ & $0.7170 \pm 0.0030$ & $102990 \pm 850$ & $102990 \pm 850$ & $204.0 \pm 4.0$ & $102930 \pm 850$ & 47.4 \\
\hline EXC3-6.8 & $1415.0 \pm 2.0$ & $400 \pm 10$ & $41590 \pm 850$ & $150.0 \pm 1.0$ & $0.7160 \pm 0.0010$ & $103160 \pm 360$ & $103150 \pm 360$ & $201.0 \pm 2.0$ & $103090 \pm 360$ & 68.0 \\
\hline EXC3-8.0 & $1060.0 \pm 2.0$ & $182 \pm 4$ & $69300 \pm 1480$ & $153.0 \pm 2.0$ & $0.7200 \pm 0.0010$ & $103650 \pm 410$ & $103640 \pm 410$ & $205.0 \pm 2.0$ & $103580 \pm 410$ & 81.9 \\
\hline EXC3-9.0 & $1259.0 \pm 1.0$ & $188 \pm 4$ & $79020 \pm 1680$ & $148.0 \pm 1.0$ & $0.7160 \pm 0.0010$ & $103650 \pm 370$ & $103640 \pm 370$ & $198.0 \pm 2.0$ & $103580 \pm 370$ & 90.0 \\
\hline EXC3-11.5 & $1084.0 \pm 1.0$ & $102 \pm 2$ & $126160 \pm 2960$ & $150.0 \pm 2.0$ & $0.7190 \pm 0.0010$ & $103890 \pm 370$ & $103890 \pm 370$ & $201.0 \pm 2.0$ & $103830 \pm 370$ & 115.0 \\
\hline EXC3-12.8 & $976.0 \pm 1.0$ & $62 \pm 2$ & $185870 \pm 5760$ & $147.0 \pm 2.0$ & $0.7180 \pm 0.0010$ & $104160 \pm 380$ & $104160 \pm 380$ & $197.0 \pm 2.0$ & $104100 \pm 380$ & 127.7 \\
\hline EXC3-14.3 & $1331.0 \pm 2.0$ & $158 \pm 3$ & $99650 \pm 2190$ & $145.0 \pm 1.0$ & $0.7170 \pm 0.0010$ & $104340 \pm 370$ & $104340 \pm 370$ & $195.0 \pm 2.0$ & $104280 \pm 370$ & 142.8 \\
\hline EXC3-15.4 & $1124.0 \pm 1.0$ & $37 \pm 1$ & $359130 \pm 12980$ & $149.0 \pm 2.0$ & $0.7200 \pm 0.0010$ & $104420 \pm 370$ & $104420 \pm 370$ & $200.0 \pm 2.0$ & $104360 \pm 370$ & 153.9 \\
\hline EXC3-16.1 & $1393.0 \pm 2.0$ & $41 \pm 1$ & $401510 \pm 14620$ & $148.0 \pm 2.0$ & $0.7210 \pm 0.0010$ & $104620 \pm 390$ & $104620 \pm 390$ & $199.0 \pm 2.0$ & $104560 \pm 390$ & 161.4 \\
\hline EXC3-17.1 & $1266.0 \pm 2.0$ & $550 \pm 10$ & $27210 \pm 550$ & $144.0 \pm 2.0$ & $0.7190 \pm 0.0010$ & $104860 \pm 430$ & $104850 \pm 430$ & $193.0 \pm 2.0$ & $104790 \pm 430$ & 170.9 \\
\hline EXC3-17.9 & $1533.0 \pm 2.0$ & $520 \pm 10$ & $34820 \pm 710$ & $139.0 \pm 2.0$ & $0.7180 \pm 0.0010$ & $105660 \pm 440$ & $105650 \pm 440$ & $187.0 \pm 2.0$ & $105590 \pm 440$ & 179.0 \\
\hline EXC3-18.7 & $1176.0 \pm 2.0$ & $1000 \pm 20$ & $14040 \pm 280$ & $141.0 \pm 2.0$ & $0.7210 \pm 0.0020$ & $105890 \pm 480$ & $105870 \pm 480$ & $190.0 \pm 2.0$ & $105810 \pm 480$ & 187.8 \\
\hline EXC3-20.0 & $123.1 \pm 0.1$ & $132 \pm 3$ & $11140 \pm 220$ & $141.0 \pm 2.0$ & $0.7220 \pm 0.0010$ & $106070 \pm 380$ & $106040 \pm 380$ & $190.0 \pm 2.0$ & $105980 \pm 380$ & 200.0 \\
\hline EXC3-20.9 & $1372.0 \pm 2.0$ & $430 \pm 10$ & $37540 \pm 763$ & $139.0 \pm 1.0$ & $0.7200 \pm 0.0010$ & $105950 \pm 370$ & $105940 \pm 370$ & $188.0 \pm 2.0$ & $105880 \pm 370$ & .7 \\
\hline EXC3-21.8 & $1254.0 \pm 1.0$ & $183 \pm 4$ & $81860 \pm 1730$ & $145.0 \pm 2.0$ & $0.7260 \pm 0.0010$ & $106270 \pm 410$ & $106270 \pm 410$ & $196.0 \pm 2.0$ & $106210 \pm 410$ & 217 \\
\hline EXC3-22.6 & $1393.0 \pm 2.0$ & $990 \pm 20$ & $16880 \pm 340$ & $147.0 \pm 2.0$ & $0.7290 \pm 0.0010$ & $106830 \pm 410$ & $106810 \pm 410$ & $198.0 \pm 2.0$ & $106750 \pm 410$ & 225.9 \\
\hline EXC3-23.4 & $2979.0 \pm 5.0$ & $790 \pm 20$ & $5580 \pm 930$ & $147.0 \pm 2.0$ & $0.7300 \pm 0.0020$ & $106920 \pm 460$ & $106910 \pm 460$ & $199.0 \pm 2.0$ & $0 \pm 460$ & 233.9 \\
\hline EXC3-24.3 & $1061.0 \pm 1.0$ & $250 \pm 10$ & $51260 \pm 1070$ & $145.0 \pm 2.0$ & $0.7300 \pm 0.0010$ & $107520 \pm 420$ & $107520 \pm 420$ & $196.0 \pm 2.0$ & $107460 \pm 420$ & 243.0 \\
\hline EXC3-24.6 & $895.0 \pm 2.0$ & $70 \pm 10$ & $144720 \pm 13520$ & $145.0 \pm 3.0$ & $0.7330 \pm 0.0030$ & $108050 \pm 880$ & $108050 \pm 880$ & $197.0 \pm 3.0$ & $107990 \pm 880$ & 246.2 \\
\hline EXC3-25.5 & $1233.0 \pm 2.0$ & $92 \pm 2$ & $160930 \pm 4020$ & $141.0 \pm 2.0$ & $0.7310 \pm 0.0010$ & $108170 \pm 410$ & $108170 \pm 410$ & $192.0 \pm 2.0$ & $108110 \pm 410$ & 254.7 \\
\hline EXC3-26.0 & $1009.0 \pm 2.0$ & $370 \pm 10$ & $32830 \pm 670$ & $146.0 \pm 2.0$ & $0.7340 \pm 0.0020$ & $108170 \pm 490$ & $108160 \pm 490$ & $198.0 \pm 2.0$ & $108100 \pm 490$ & 260.0 \\
\hline EXC3-26.8 & $1076.0 \pm 1.0$ & $830 \pm 20$ & $15800 \pm 320$ & $147.0 \pm 2.0$ & $0.7350 \pm 0.0010$ & $108290 \pm 400$ & $108270 \pm 400$ & $200.0 \pm 2.0$ & $108210 \pm 400$ & 267.9 \\
\hline EXC3-27.8 & $941.0 \pm 2.0$ & $73 \pm 2$ & $155730 \pm 3850$ & $146.0 \pm 2.0$ & $0.7380 \pm 0.0020$ & $109030 \pm 500$ & $109030 \pm 500$ & $199.0 \pm 2.0$ & $108970 \pm 500$ & 278.0 \\
\hline EXC3-28.9 & $763.0 \pm 2.0$ & $1050 \pm 10$ & $8850 \pm 90$ & $144.0 \pm 2.0$ & $0.7400 \pm 0.0030$ & $109950 \pm 920$ & $109910 \pm 920$ & $197.0 \pm 3.0$ & $109850 \pm 920$ & 290.4 \\
\hline EXC3-29.5 & $872.0 \pm 1.0$ & $124 \pm 3$ & $86130 \pm 1980$ & $144.0 \pm 1.0$ & $0.7400 \pm 0.0010$ & $110060 \pm 400$ & $110060 \pm 400$ & $197.0 \pm 2.0$ & $110000 \pm 400$ & \\
\hline EXC4-12.4 & $877.0 \pm 1.0$ & $115 \pm 3$ & $76440 \pm 1830$ & $174.0 \pm 2.0$ & $0.6060 \pm 0.0010$ & $77510 \pm 230$ & $77510 \pm 230$ & $216.0 \pm 2.0$ & $77450 \pm 230$ & 122.3 \\
\hline EXC4-15.2 & $1213.0 \pm 2.0$ & $470 \pm 10$ & $25730 \pm 520$ & $171.0 \pm 2.0$ & $0.6090 \pm 0.0010$ & $78290 \pm 250$ & $78280 \pm 250$ & $214.0 \pm 2.0$ & $78220 \pm 250$ & 3 \\
\hline EXC4-18.0 & $830.0 \pm 1.0$ & $2500 \pm 50$ & $3350 \pm 70$ & $177.0 \pm 2.0$ & $0.6130 \pm 0.0010$ & $78460 \pm 300$ & $78390 \pm 310$ & $221.0 \pm 2.0$ & $78330 \pm 310$ & \\
\hline EXC4-21.4 & $793.0 \pm 1.0$ & $100 \pm 2$ & $82720 \pm 2000$ & $162.0 \pm 2.0$ & $0.6170 \pm 0.0010$ & $80770 \pm 270$ & $80770 \pm 270$ & $204.0 \pm 2.0$ & $80710 \pm 270$ & \\
\hline EXC4-24.1 & $694.0 \pm 2.0$ & $260 \pm 10$ & $27210 \pm 680$ & $162.0 \pm 3.0$ & $0.6260 \pm 0.0030$ & $82530 \pm 660$ & $82520 \pm 660$ & $205.0 \pm 4.0$ & $82460 \pm 660$ & \\
\hline EXC4-25.1 & $832.0 \pm 1.0$ & $740 \pm 20$ & $11560 \pm 230$ & $160.0 \pm 1.0$ & $0.6270 \pm 0.0010$ & $82900 \pm 270$ & $82880 \pm 270$ & $202.0 \pm 2.0$ & $82820 \pm 270$ & 250.8 \\
\hline EXC4-25.5 & $771.0 \pm 1.0$ & $115 \pm 3$ & $74850 \pm 1710$ & $143.0 \pm 2.0$ & $0.6750 \pm 0.0010$ & $94880 \pm 370$ & $94880 \pm 370$ & $187.0 \pm 2.0$ & $94820 \pm 370$ & 255.3 \\
\hline EXC4-26.8 & $1008.0 \pm 1.0$ & $640 \pm 10$ & $17640 \pm 360$ & $142.0 \pm 2.0$ & $0.6780 \pm 0.0010$ & $95820 \pm 320$ & $95810 \pm 320$ & $186.0 \pm 2.0$ & $95750 \pm 320$ & 267.3 \\
\hline EXC4-29.1 & $782.0 \pm 1.0$ & $22 \pm 1$ & $401480 \pm 23250$ & $140.0 \pm 2.0$ & $0.6840 \pm 0.0010$ & $97530 \pm 390$ & $97530 \pm 390$ & $184.0 \pm 2.0$ & $97470 \pm 390$ & 291.8 \\
\hline EXC4-30.5 & $830.0 \pm 1.0$ & $400 \pm 10$ & $23810 \pm 490$ & $140.0 \pm 1.0$ & $0.6920 \pm 0.0010$ & $99320 \pm 340$ & $99310 \pm 340$ & $185.0 \pm 2.0$ & $99250 \pm 340$ & 305.0 \\
\hline EXC4-32.0 & $1025.0 \pm 1.0$ & $142 \pm 3$ & $82600 \pm 1900$ & $131.0 \pm 1.0$ & $0.6940 \pm 0.0010$ & $101220 \pm 360$ & $101210 \pm 360$ & $174.0 \pm 2.0$ & $101150 \pm 360$ & 319.8 \\
\hline EXC4-33.0 & $958.0 \pm 1.0$ & $160 \pm 3$ & $69050 \pm 1490$ & $134.0 \pm 2.0$ & $0.7000 \pm 0.0010$ & $102020 \pm 380$ & $102020 \pm 380$ & $179.0 \pm 2.0$ & $101960 \pm 380$ & 330.0 \\
\hline EXC4-33.1 & $853.0 \pm 2.0$ & $16 \pm 10$ & $608880 \pm 264500$ & $141.0 \pm 2.0$ & $0.7070 \pm 0.0030$ & $102510 \pm 790$ & $102510 \pm 790$ & $189.0 \pm 3.0$ & $102450 \pm 790$ & 334.8 \\
\hline EXC4-34.1 & $794.0 \pm 1.0$ & $250 \pm 10$ & $38060 \pm 790$ & $143.0 \pm 2.0$ & $0.7140 \pm 0.0010$ & $103780 \pm 410$ & $103770 \pm 410$ & $192.0 \pm 2.0$ & $103710 \pm 410$ & 344.0 \\
\hline EXC4-35.5 & $787.0 \pm 1.0$ & $320 \pm 10$ & $29010 \pm 590$ & $141.0 \pm 1.0$ & $0.7150 \pm 0.0010$ & $104530 \pm 360$ & $104520 \pm 360$ & $189.0 \pm 2.0$ & $104460 \pm 360$ & 355.3 \\
\hline EXC4-36.0 & $965.0 \pm 1.0$ & $1420 \pm 30$ & $8050 \pm 160$ & $142.0 \pm 2.0$ & $0.7190 \pm 0.0010$ & $105260 \pm 350$ & $105220 \pm 350$ & $191.0 \pm 2.0$ & $105160 \pm 350$ & \\
\hline EXC4-37.0 & $942.0 \pm 1.0$ & $540 \pm 10$ & $20850 \pm 420$ & $142.0 \pm 1.0$ & $0.7210 \pm 0.0010$ & $105730 \pm 360$ & $105710 \pm 360$ & $191.0 \pm 2.0$ & $105650 \pm 360$ & 369.8 \\
\hline EXC4-37.5 & $911.0 \pm 2.0$ & $210 \pm 10$ & $51130 \pm 1610$ & $146.0 \pm 2.0$ & $0.7280 \pm 0.0030$ & $106740 \pm 820$ & $106730 \pm 820$ & $198.0 \pm 3.0$ & $106670 \pm 820$ & 378.5 \\
\hline EXC4-38.4 & $896.0 \pm 1.0$ & $2350 \pm 50$ & $4600 \pm 90$ & $147.0 \pm 2.0$ & $0.7300 \pm 0.0010$ & $107200 \pm 370$ & $107140 \pm 380$ & $198.0 \pm 2.0$ & $107080 \pm 380$ & 383.8 \\
\hline $\mathrm{SCH} 7$ & & & & & & & & & & \\
\hline $\mathrm{SCH} 7-1.2$ & $130.1 \pm 0.1$ & $1820 \pm 40$ & $990 \pm 20$ & $262.0 \pm 2.0$ & $0.8390 \pm 0.0020$ & $112950 \pm 470$ & $112650 \pm 510$ & $360.0 \pm 3.0$ & $112590 \pm 510$ & 12.0 \\
\hline $\mathrm{SCH} 7-2.4$ & $110.3 \pm 0.1$ & $2250 \pm 50$ & $680 \pm 10$ & $252.0 \pm 2.0$ & $0.8370 \pm 0.0020$ & $114230 \pm 480$ & $113790 \pm 570$ & $348.0 \pm 3.0$ & $113730 \pm 570$ & 24.0 \\
\hline SCH7-2.6 & $113.4 \pm 0.1$ & $3040 \pm 60$ & $510 \pm 10$ & $248.0 \pm 2.0$ & $0.8310 \pm 0.0020$ & $113720 \pm 500$ & $113130 \pm 650$ & $341.0 \pm 3.0$ & $113070 \pm 650$ & 26.3 \\
\hline SCH7-3.0 & $133.5 \pm 0.1$ & $5250 \pm 110$ & $350 \pm 10$ & $252.0 \pm 2.0$ & $0.8400 \pm 0.0020$ & $114980 \pm 520$ & $114120 \pm 790$ & $348.0 \pm 3.0$ & $114060 \pm 790$ & 30.0 \\
\hline $\mathrm{SCH} 7-3.5$ & $118.7 \pm 0.1$ & $340 \pm 10$ & $4850 \pm 100$ & $261.0 \pm 2.0$ & $0.8470 \pm 0.0020$ & $114950 \pm 530$ & $114890 \pm 530$ & $361.0 \pm 3.0$ & $114830 \pm 530$ & 34.5 \\
\hline SCH7-4.5 & $119.6 \pm 0.1$ & $59 \pm 2$ & $28310 \pm 900$ & $268.0 \pm 2.0$ & $0.8540 \pm 0.0020$ & $115420 \pm 530$ & $115410 \pm 530$ & $371.0 \pm 2.0$ & $115350 \pm 530$ & 45.0 \\
\hline SCH7-5.5 & $156.7 \pm 0.2$ & $94 \pm 2$ & $23430 \pm 550$ & $266.0 \pm 2.0$ & $0.8520 \pm 0.0020$ & $115210 \pm 470$ & $115200 \pm 470$ & $368.0 \pm 3.0$ & $115140 \pm 470$ & 54.8 \\
\hline SCH7-5.9 & $111.2 \pm 0.1$ & $67 \pm 2$ & $23260 \pm 590$ & $263.0 \pm 2.0$ & $0.8520 \pm 0.0020$ & $115750 \pm 510$ & $115730 \pm 510$ & $365.0 \pm 3.0$ & $115670 \pm 510$ & 59.0 \\
\hline SCH7-6.1 & $138.0 \pm 0.1$ & $220 \pm 10$ & $9000 \pm 200$ & $267.0 \pm 2.0$ & $0.8520 \pm 0.0020$ & $115020 \pm 510$ & $114990 \pm 510$ & $369.0 \pm 3.0$ & $114930 \pm 510$ & 61.0 \\
\hline $\mathrm{SCH} 7-6.5$ & $157.4 \pm 0.2$ & $116 \pm 3$ & $19160 \pm 420$ & & $0.8530 \pm 0.0020$ & $115460 \pm 470$ & & $369.0 \pm 3.0$ & $115390 \pm 470$ & \\
\hline $\mathrm{SCH} 7-7.0$ & $139.0 \pm 0.1$ & $370 \pm 10$ & $5330 \pm 110$ & $268.0 \pm 2.0$ & $0.8530 \pm 0.0020$ & $115090 \pm 450$ & $115030 \pm 450$ & $371.0 \pm 2.0$ & $114970 \pm 450$ & 70.0 \\
\hline SCH7-7.4 & $165.2 \pm 0.2$ & $181 \pm 4$ & $12960 \pm 270$ & $273.0 \pm 2.0$ & $0.8620 \pm 0.0020$ & $116530 \pm 490$ & $116500 \pm 490$ & $379.0 \pm 3.0$ & $116440 \pm 490$ & 74.0 \\
\hline SCH7-8.0 & $136.3 \pm 0.1$ & $230 \pm 10$ & $8400 \pm 180$ & $265.0 \pm 2.0$ & $0.8540 \pm 0.0020$ & $115910 \pm 500$ & $115870 \pm 500$ & $368.0 \pm 2.0$ & $115810 \pm 500$ & 80.3 \\
\hline SCH7-9.0 & $160.5 \pm 0.2$ & $790 \pm 20$ & $2890 \pm 60$ & $264.0 \pm 2.0$ & $0.8580 \pm 0.0020$ & $116900 \pm 500$ & $116790 \pm 510$ & $368.0 \pm 3.0$ & $116730 \pm 510$ & 90.0 \\
\hline SCH7-9.7 & $165.7 \pm 0.1$ & $320 \pm 10$ & $7220 \pm 150$ & $260.0 \pm 2.0$ & $0.8570 \pm 0.0010$ & $117470 \pm 440$ & $117430 \pm 440$ & $363.0 \pm 2.0$ & $117370 \pm 440$ & 96.8 \\
\hline $\mathrm{SCH} 7-10.3$ & $201.0 \pm 0.2$ & $850 \pm 20$ & $3340 \pm 70$ & $260.0 \pm 2.0$ & $0.8570 \pm 0.0020$ & $117500 \pm 480$ & $117410 \pm 480$ & $362.0 \pm 3.0$ & $117350 \pm 480$ & 102.8 \\
\hline $\mathrm{SCH} 7-11.0$ & $139.6 \pm 0.1$ & $143 \pm 3$ & $13650 \pm 300$ & $248.0 \pm 2.0$ & $0.8490 \pm 0.0020$ & $117720 \pm 500$ & $117700 \pm 500$ & $346.0 \pm 2.0$ & $117640 \pm 500$ & 110.0 \\
\hline SCH7-11.3 & $147.5 \pm 0.2$ & $143 \pm 3$ & $14330 \pm 320$ & $239.0 \pm 2.0$ & $0.8430 \pm 0.0020$ & $118000 \pm 550$ & $117980 \pm 550$ & $334.0 \pm 3.0$ & $117920 \pm 550$ & 113.3 \\
\hline SCH7-11.4 & $117.2 \pm 0.1$ & $1350 \pm 30$ & $1220 \pm 30$ & $242.0 \pm 2.0$ & $0.8500 \pm 0.0020$ & $119060 \pm 490$ & $118810 \pm 520$ & $339.0 \pm 2.0$ & $118750 \pm 520$ & 114.0 \\
\hline
\end{tabular}

a $\delta^{234} \mathrm{U}=\left(\left[{ }^{234} \mathrm{U} / 238 \mathrm{U}\right]_{\text {activity }}-1\right) \times 1000 . \delta^{234} \mathrm{U}_{\text {initial }}$ was calculated based on ${ }^{230} \mathrm{Th}$ age (T), i.e., $\delta^{234} \mathrm{U}_{\text {initial }}=\delta^{234} \mathrm{U}_{\text {measured }} \times e^{\lambda 234 x T}$. b Corrected ${ }^{230} \mathrm{Th}$ ages assume the initial ${ }^{230} \mathrm{Th} /{ }^{232} \mathrm{Th}$ atomic ratio of $4.4 \pm 2.2 \times 10^{-6}$. Those are the values for a material at secular equilibrium, with the bulk earth ${ }^{232} \mathrm{Th} /{ }^{238} \mathrm{U}$ value of 3.8 . The errors are arbitrarily assumed to be $50 \%$. ${ }^{\mathrm{c}}$ a BP stands for "years Before Present" where the present is defined as the year 1950 A.D. ${ }^{\mathrm{d}}$ DFT stands for "Distance From Top" of the stalagmite. 


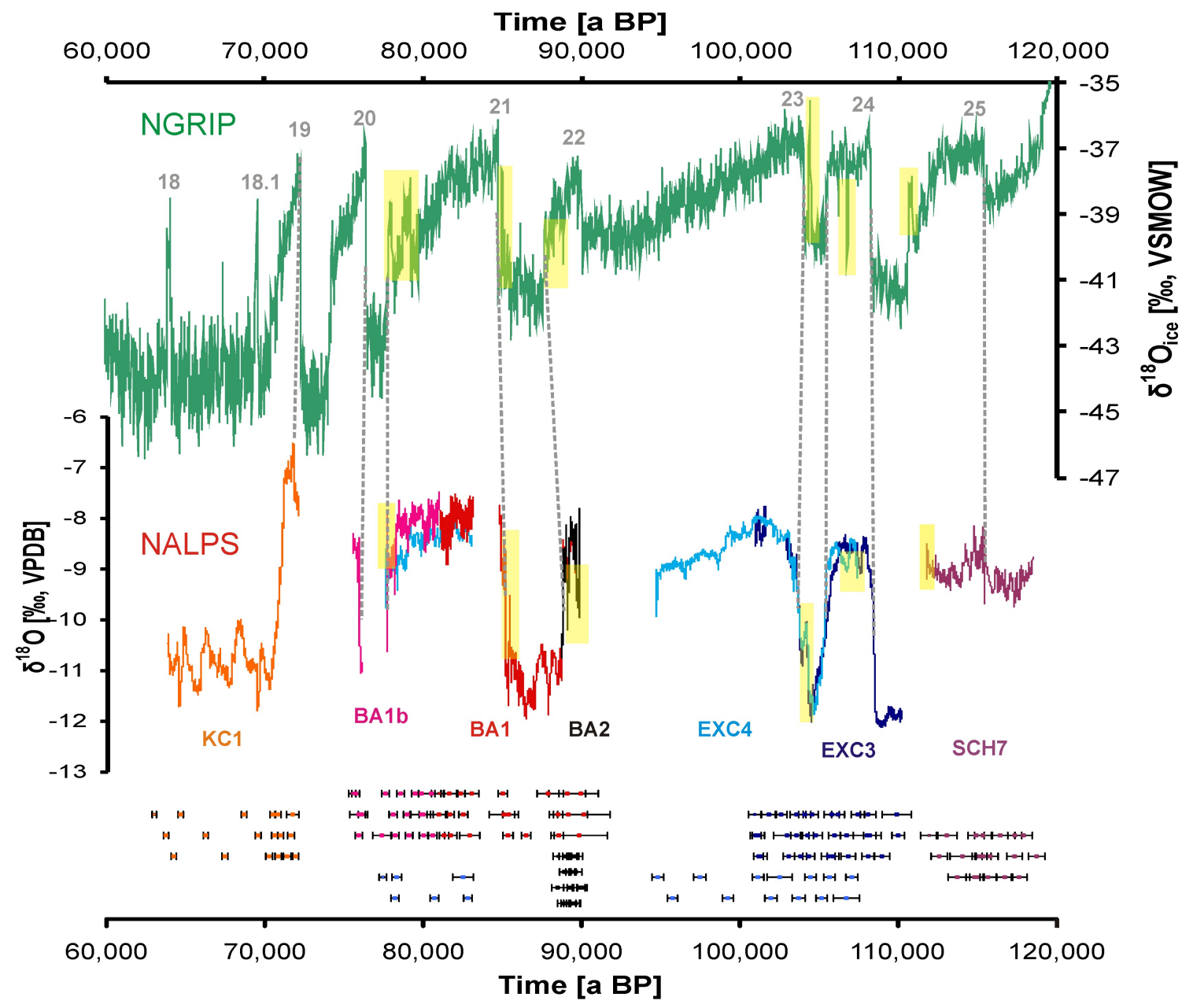

Fig. 2. The NALPS record (consisting of seven stalagmites) compared to NGRIP plotted on the GICC05modelext timescale in the interval from 120 to $60 \mathrm{ka}$. Individual U-Th ages and associated $2 \sigma$-uncertainties are plotted at the bottom. The grey, dashed lines connect the mid-points of major D-O transitions or isotopic maxima. The recurrent warm Greenland Interstadials of the D-O cycles are indicated by grey numbers. Short-lived warming and cooling events (sub-D-O events) are highlighted by the yellow rectangles. NALPS confirms the Greenland chronology for the early part of the Last Glacial period, but suggests younger ages of rapid stadial and interstadial transitions between ca. 106 and $60 \mathrm{ka}$, and a longer duration of the stadial following GI 22.

reflect the $\mathrm{O}$ isotopic composition of regional meteoric precipitation and in the Alps this variable is strongly correlated with air temperature (Rozanski et al., 1992; Humer et al., 1995; Kaiser et al., 2001). Moreover, the temperature of carbonate precipitation in the cave is directly related to the outside air temperature and determines the $\mathrm{O}$ isotopic fractionation between drip water and speleothem calcite (Friedman and O'Neil, 1977; Kim and O'Neil, 1997; Lachniet, 2009). Therefore, the air temperature has a major, combined effect on the speleothem $\mathrm{O}$ isotopic composition. Seasonality, however, might also affect the isotope signal in the Alps (Humer et al., 1995), as the seasonal amplitude in $\delta^{18} \mathrm{O}$ of meteoric precipitation is around $10 \%$ (IAEA/WMO, 2010). The distribution of precipitation during the warm and cold season might thus exert a major influence on the overall isotopic composition. This could partially explain the large shifts at the D-O transitions. Major changes in the source region of precipitation, e.g. Atlantic vs. Mediterranean (cf. Spötl et al., 2010), are considered negligible on the northern rim of the Alps, whereas changes in the trajectories of moisture across the European continent might have some influence (cf. Sodemann and Zubler, 2010). Replication of intervals in different stalagmite samples suggests neither cave-specific nor drip site-specific effects had a major influence on the $\mathrm{O}$ isotopic composition. The unsaturated zone above the studied caves ranges from ca. 10 to $200 \mathrm{~m}$ allowing for fast transmission of the meteoric $\mathrm{O}$ isotopic signal by the seepage water.

A synchronous occurrence of the rapid climate changes recorded in Greenland and NALPS is a reasonable assumption given the location of the selected cave sites at the northern, Atlantic-exposed rim of the Alps. The records reveal eye-catching similarities in the detailed pattern of individual 
D-O events between the Alps and Greenland. Most of the events are very similar in the duration and relative amplitudes at both locations. Moreover, the details recorded both in the ice and stalagmite isotope curves, e.g. the short-lived subD-O warming and cooling events (see Sect. 6), are a strong argument for synchronicity between Greenland and Europe. An asynchronous or systematically different climate evolution in the two regions is clearly not supported by the data and we therefore do not discuss potential leads and lags or systematic offsets. The observed differences in the timing of the D-O events most likely originate from the different chronologies, i.e. a problem that can be solved within the dating uncertainties of the Greenland and NALPS records. For that reason we concentrate on discussing these chronological issues.

In general, the rapid transitions of 1 to $4.5 \%$ in $\delta^{18} \mathrm{O}$ took place within decades (central shift) to several centuries (whole transition) in our samples (Table 2). The central shift encompasses the abrupt and also largest part of the amplitude of a D-O transition. The whole transition also includes the less abrupt, i.e. more gradual flanks and the entire amplitude of a transition. In particular, the transition from Marine Isotope Stage (MIS) 5 to 4, i.e. the D-O 19 cooling, lasted $400 \mathrm{yr}$ in its central portion (2.8\%o shift) and $950 \mathrm{yr}$ for the whole transition (4.3\%o; Fig. 3; stalagmite KC1). The DO 20 warming lasted $110 \mathrm{yr}(2.6 \%$, stalagmite BA1b) and D-O 21 cooling $20 \mathrm{yr}$ (2.7\%o, BA1b; the event, however, is not recorded completely). The central portion of the DO 21 rapid warming $(2.3 \%$; BA1) lasted $60 \mathrm{yr}$, while the whole transition took place within $550 \mathrm{yr}(4.0 \%$, BA1). The cooling of D-O 22 occurred within $60 \mathrm{yr}$ (2.2\%o; stalagmite BA2) and the cooling of D-O 24 lasted $120 \mathrm{yr}$ in its central part $(1.2 \%$ ) and $1050 \mathrm{yr}$ for the whole transition (3.3\%o, EXC4). Stalagmite EXC3 recorded the warmings of D-O 23 and 24: the central shifts of $2.2 \%$ each occurred within 340 and $170 \mathrm{yr}$, respectively, while the whole transitions of 3.6 and $3.5 \%$ lasted 1400 and $590 \mathrm{yr}$, respectively. The minor D-O 25 warming $(1.5 \%$ ) lasted $20 \mathrm{yr}$ in our record (Fig. 3; stalagmite $\mathrm{SCH} 7$ ).

\section{Chronological implications}

Significant chronological implications arise for the current Greenland ice-core timescale. In the following discussion we exclusively use the updated timescale for the NGRIP ice core (GICC05modelext; Wolff et al., 2010). The MIS 5/4 transition, i.e. the D-O 19 cooling transition, is recorded in stalagmite $\mathrm{KC} 1$ in great detail (Fig. 2). D-O 19 started with a rapid increase towards positive $\mathrm{O}$ isotope values, followed by a gradual cooling over several centuries and a rapid drop towards negative values into MIS 4, which reflects the typical pattern associated with D-O fluctuations (e.g. Rahmstorf, 2002; Peavoy and Franzke, 2010). The GI 19 isotopic maximum occurred at $71690 \pm 220$ a BP (average $2 \sigma$-error at the isotope maximum), compared to 72090 a BP in the NGRIP record (Fig. 2). The two tie points (isotopic maxima) thus exhibit a difference of $400 \mathrm{yr}$, i.e. NALPS suggests a younger age (Fig. 2 and Table 2). In the East Asian cave records (e.g. Wang et al., 2001, 2008) the MIS5/4 transition is not as pronounced as in the NALPS record. D-O transitions in the monsoonal records are generally more gradual and the cold/dry stadials are smoother than in alpine samples. Going further back in time, the rapid transition into GI 20 is constrained to $75860 \pm 300$ a (mid-point of the transition; Fig. 2) compared to $76410 \mathrm{a}$ in the ice-core record, i.e. NALPS is younger by $550 \mathrm{yr}$. The mid-point of the transition from GI 21 to the subsequent stadial is located at $77580 \pm 240 \mathrm{a}$ in NALPS and at 77795 a in NGRIP, i.e. a difference of $215 \mathrm{yr}$.

Regarding the long stadial following GI 22 , there is a mismatch between the ice core and the speleothem record (Fig. 2). The transition into GI 21 (mid-point) is constrained to $84730 \mathrm{a}$ in NGRIP and to $85030 \pm 410 \mathrm{a}$ in stalagmite BA1, i.e. NALPS is only $300 \mathrm{yr}$ older, which is within the error of the associated U-Th ages. The transition from GI 22 into the stadial, however, reveals an age of $87630 \mathrm{a}$ in NGRIP versus $88690 \pm 330$ a in NALPS, i.e. this is the only transition in the first half of the Last Glacial, where NALPS suggests a significantly older age than Greenland (by $1060 \mathrm{yr}$ ). The sharp transition into the stadial following GI 22 is recorded in the two stalagmites BA2 and BA1, but is only constrained precisely in BA2 (Fig. 2). For that reason, the age model of BA1 in the overlapping section was adjusted to the age model of BA2 using the software AnalySeries (Paillard et al., 1996). Based on several precise U-Th ages, however, there is a distinct discrepancy in the duration of the stadial: it was approximately $2900 \mathrm{yr}$ long based on the current ice core chronology, while it lasted ca. $3650 \mathrm{yr}$ according to the NALPS record. A relatively long stadial following GI 22 is also supported by East Asian stalagmite records (e.g. Sanbao Cave; Wang et al., 2008).

The D-O transition from into GI 23 is constrained to 103995 a in NGRIP compared to $103550 \pm 375$ a in NALPS, i.e. the latter is $445 \mathrm{yr}$ younger (Fig. 2). The rapid cooling from GI 24 to the subsequent stadial occurred at $105410 \mathrm{a}$ in the ice core and at $105210 \pm 450 \mathrm{a}$ in stalagmite EXC4, i.e. a difference of $200 \mathrm{yr}$. The latter transition is also recorded in stalagmite EXC3, although the isotope drop is less pronounced there. A stalagmite record from Corchia Cave, Italy (Drysdale et al., 2007) also covers the time interval from 118 to $96 \mathrm{ka}$. In this record, the stadial following GI 24 appears rather long and the transition into GI 23 occurs somewhat late compared to NALPS, although it is within the $2 \sigma$-age uncertainty (0.8-1.0 kyr; Drysdale et al., 2007). The progression of GI 23 is very similar in both speleothem records, i.e. showing a long-term cooling trend. The mid-point of the transition into GI 24 is constrained to $108250 \mathrm{a}$ in NGRIP and to $108300 \pm 450 \mathrm{a}$ in NALPS; the small discrepancy of $50 \mathrm{yr}$ is well within the errors of the U-Th age model. Similarly, the transition into GI 25 is constrained to 115350 a 
Table 2. Overview on timing, duration and amplitude of D-O transitions and short-lived sub-D-O events.

\begin{tabular}{|c|c|c|c|c|c|c|c|c|}
\hline \multirow[t]{2}{*}{ Event $^{\mathrm{a}}$} & \multicolumn{4}{|c|}{$\begin{array}{l}\text { Timing }^{\mathrm{b}} \\
{[\mathrm{aBP}]}\end{array}$} & \multicolumn{2}{|c|}{$\begin{array}{c}\text { Duration of transition }^{\mathrm{c}} \\
{[\mathrm{yr}]}\end{array}$} & \multicolumn{2}{|c|}{$\begin{array}{l}\text { Amplitude } \\
{[\% o]}\end{array}$} \\
\hline & NALPS & Error, $2 \sigma[\mathrm{a}]$ & NGRIP & Difference [yr] & Central & Whole & Central & Whole \\
\hline D-O 19 cooling, MIS5/4 & & & & & 400 & 950 & 2.8 & 4.3 \\
\hline D-O 19 isotopic max. & 71690 & \pm 220 & 72090 & -400 & & & & \\
\hline D-O 20 warming & 75860 & \pm 300 & 76410 & -550 & & 110 & & 2.6 \\
\hline D-O 21 cooling & 77580 & \pm 240 & 77795 & -215 & & 20 & & 2.7 \\
\hline D-O 21 warming & 85030 & \pm 410 & 84730 & +300 & 60 & 550 & 2.3 & 4.0 \\
\hline D-O 22 cooling & 88690 & \pm 330 & 87630 & +1060 & & 60 & & 2.2 \\
\hline D-O 23 warming & 103550 & \pm 375 & 103995 & -445 & 340 & 1400 & 2.2 & 3.6 \\
\hline D-O 24 cooling & 105210 & \pm 450 & 105410 & -200 & 120 & 1050 & 1.2 & 3.3 \\
\hline D-O 24 warming & 108300 & \pm 450 & 108250 & +50 & 170 & 590 & 2.2 & 3.5 \\
\hline \multirow[t]{2}{*}{ D-O 25 warming } & 115320 & \pm 500 & 115350 & -30 & & 20 & & 1.5 \\
\hline & \multicolumn{2}{|r|}{$\begin{array}{l}\text { From } \\
{[\mathrm{a} \mathrm{BP}]}\end{array}$} & \multicolumn{2}{|r|}{$\begin{array}{c}\text { To } \\
{[\mathrm{a} \mathrm{BP}]}\end{array}$} & \multicolumn{2}{|c|}{$\begin{array}{l}\text { Duration of event } \\
{[\mathrm{yr}]}\end{array}$} & \multicolumn{2}{|c|}{$\begin{array}{c}\text { Increase/Decrease }^{\mathrm{e}} \\
{[\% o]}\end{array}$} \\
\hline GI 21 rebound & \multicolumn{2}{|r|}{77730} & \multicolumn{2}{|r|}{77580} & \multicolumn{2}{|c|}{150} & \multicolumn{2}{|c|}{1.2} \\
\hline GI 21 precursor I & \multicolumn{2}{|r|}{85440} & \multicolumn{2}{|r|}{85250} & \multicolumn{2}{|c|}{190} & \multicolumn{2}{|c|}{$1.7 /-2.2$} \\
\hline GI 21 precursor II & \multicolumn{2}{|r|}{85070} & \multicolumn{2}{|r|}{84970} & \multicolumn{2}{|c|}{100} & \multicolumn{2}{|c|}{$2.4 /-1.0$} \\
\hline GI 22 transient cooling I & \multicolumn{2}{|r|}{89700} & \multicolumn{2}{|r|}{89490} & \multicolumn{2}{|c|}{210} & \multicolumn{2}{|c|}{-1.8} \\
\hline GI 22 transient cooling II & \multicolumn{2}{|r|}{89130} & \multicolumn{2}{|r|}{88880} & \multicolumn{2}{|c|}{250} & \multicolumn{2}{|c|}{-2.1} \\
\hline GI 23 precursor & \multicolumn{2}{|r|}{104170} & \multicolumn{2}{|r|}{103770} & \multicolumn{2}{|c|}{400} & \multicolumn{2}{|c|}{$1.2 /-0.8$} \\
\hline GI 24 transient cooling & \multicolumn{2}{|r|}{107620} & & 107240 & & & & 0.7 \\
\hline GI 25 rebound & & 111780 & & 111600 & & & & 0.7 \\
\hline
\end{tabular}

a D-O events and short-lived sub-D-O warming (precursor and rebound) and cooling events.

b Mid-point of the respective transitions in the NALPS and NGRIP (GICC05modelext) records.

c Sharp rapid shift (central) is often flanked by more gradual progressions (whole transition) towards isotopic maxima/minima.

d Oxygen isotopic amplitude of the central and whole portion of a transition.

e Oxygen isotopic increase (positive values) and decrease (negative values) during a particular sub-D-O event.

and $115320 \pm 500 \mathrm{a}$ in NGRIP and NALPS, respectively, i.e. identical within the current dating uncertainties. Compared to NALPS, the stalagmite record from Corchia Cave shows a more gradual and relatively early transition into GI 24 and also a more gradual shift into GI 25. The latter, however, is better developed in the Italian record. In Sanbao Cave (Wang et al., 2008) the maximum of GI 25 occurred relatively late and the transition is much more gradual.

Taken together, the NALPS record suggests overall younger ages for the rapid stadial and interstadial transitions compared to the current NGRIP timescale (GICC05modelext) between 120 and $60 \mathrm{ka}$ (Table 2). This observation is consistent with East Asian stalagmite records (Xia et al., 2007), as well as with the layer-counted GICC05 ice-core timescale for the period younger than $60 \mathrm{ka}$ (Svensson et al., 2008). Moreover, stalagmites from Kleegruben Cave in the Alps also suggest a shift towards younger ages in the ss09sea timescale (Johnsen et al., 2001) in the interval of GI 15 to 14 (Spötl et al., 2006). Based on our U-Th data and age models, the shift towards younger ages is ca. 200$600 \mathrm{yr}$ in the interval 106 to $60 \mathrm{ka}$, while our data support the current Greenland ice-core timescale between 118 and
$106 \mathrm{ka}$. Notably, the timing of the rapid transitions into and out of the stadial following GI 22 (Fig. 2; Table 2) is older in NALPS than in NGRIP.

\section{Details recorded in NALPS}

A comparison of the Greenland ice-core records with NALPS reveals a great deal of agreement but also some significant differences. Next to the difference in the duration of the stadial following GI 22 in NGRIP and NALPS (see above), the short-lived D-O events 18 and 18.1 are not recorded in the $\mathrm{O}$ isotopic composition of the speleothem record (Fig. 2), although stalagmite $\mathrm{KC} 1$ is obviously sensitive to rapid climate changes (it recorded D-O 19) and the mean temporal resolution in the corresponding stalagmite section is high $(11 \mathrm{a})$. In the NGRIP record the maximum amplitude of $4.5 \%$ of D-O 18 is located at $64010 \mathrm{a}$ and the interstadial lasted from 64170 to $63810 \mathrm{a}(360 \mathrm{yr})$. The maximum amplitude of $5.2 \%$ of D-O 18.1 is located at 69510 a and this warm episode lasted $460 \mathrm{yr}(69630-69170 \mathrm{a})$. Interestingly, there is also a lack of clear evidence for D-O 18 

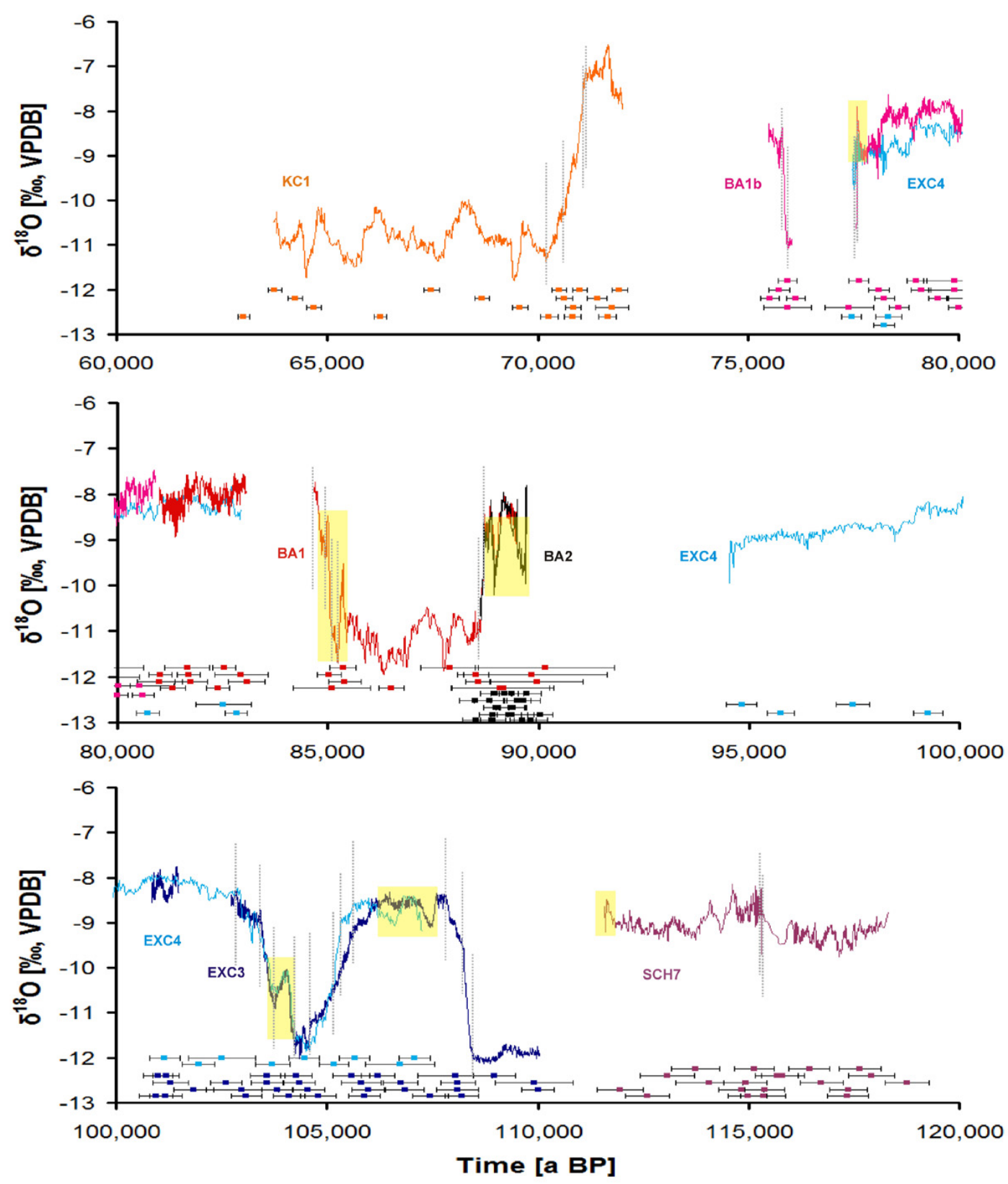

Fig. 3. Detailed structure of the NALPS record. The timing of central and whole D-O transitions (see text) is indicated by the grey, dotted lines. U-Th age data with $2 \sigma$-error bars are plotted at the bottom of the diagrams and the yellow rectangles highlight sub-D-O warming and cooling events.

in the East Asian monsoon records from Hulu and Sanbao Cave, although the temporal resolution is high enough to resolve the short interstadial during MIS 4 (Wang et al., 2001; Xia et al., 2007; Wang et al., 2008). With regard to D-O 18.1 there is evidence from new data of Hulu Cave (R. L. Edwards, unpubl. data). The observations in the Alps and East Asia provoke questions regarding the nature of some of these short-lived D-O interstadials, in particular with respect to their regional impact.
The NALPS record further resolves other short-lived details also found in the Greenland ice-core records (Fig. 3, Table 2), i.e. recurrent sub-millennial climate changes within the well-known D-O stadial and interstadial successions recently discussed by Capron et al. (2010). The intermittent climate swings consist of short and abrupt warming events preceding GI 21 and 23 (termed precursor-type events) and of rapid warming events at the end of GI 21 and 25 (termed rebound-type events). Moreover, distinct transient cooling 
events are observed during GI 22 and 24. Such superimposed and rapid Last Glacial events have not been documented outside Greenland. In the NGRIP record, a precursor-type climate event preceded GI 21 (Fig. 3, Table 2) and shows a $2.2 \%$ variation in $\delta^{18} \mathrm{O}$ of ice within $200 \mathrm{yr}$ (Capron et al., 2010). The actual onset of GI 21 coincided with a $4.2 \%$ increase in $\delta^{18} \mathrm{O}$ of ice, following a $100 \mathrm{yr}$-return to cold conditions. NALPS provides evidence of two short-lived events preceding GI 21: the first one is centred at $85360 \mathrm{a}$ and lasted for $190 \mathrm{yr}(85440-85250 \mathrm{a})$. It is characterized by a rapid increase of $1.7 \%$ and a subsequent rapid decrease of $2.2 \%$ (Fig. 3). The maximum of the second event is centred at $84990 \mathrm{a}$ and its duration was $100 \mathrm{yr}(85070-84970$ a). This event consists of a rapid $2.4 \%$ increase followed by a rapid $1.0 \%$ decrease and the final transition into GI 21 . At the end of the gradual cooling interval of GI 21 the Greenland O isotope values increased by ca. $2 \%$ in less than $100 \mathrm{yr}$, before they returned to stadial conditions (Capron et al., 2010). In the NGRIP record this rebound-type event shows two major positive peaks centred at 78970 and 77990 a (Fig. 2). The latter, shorter event at the end of GI 21 is also recorded in NALPS: the $\delta^{18} \mathrm{O}$ maximum occurred at $77590 \mathrm{a}$ and this distinct rebound of $1.2 \%$ lasted for $150 \mathrm{yr}$ (77 730-77 $580 \mathrm{a}$ ), followed by a rapid transition into the stadial. Capron et al. (2010) noted that this rebound pattern is similar to GI 22 with regard to its $\delta^{18} \mathrm{O}$ magnitude, duration and structure. In spite of this, however, it is not counted as a GI. GI 22 is characterized by two distinct cooling events centred at 88950 and $89610 \mathrm{a}$ in NALPS (recorded in stalagmite BA2). The $\mathrm{O}$ isotope values show decreases of 2.1 and $1.8 \%$ and the anomalies lasted for $250 \mathrm{yr}$ (89 130-88 880 a) and $210 \mathrm{yr}(89700-89490 \mathrm{a})$, respectively. The younger of the two events is also recorded in stalagmite BA1, although the associated U-Th age errors are significantly larger. There is also evidence for these cooling anomalies during GI 22 in the NGRIP record (Fig. 2).

Another precursor-type structure is evident immediately prior to GI 23 in Greenland: $\delta^{18} \mathrm{O}$ increased by $3.8 \%$ within $130 \mathrm{yr}$ and subsequently dropped by $3.6 \%$ in $100 \mathrm{yr}$. The latter transition back to stadial conditions lasted for $300 \mathrm{yr}$ before the $\delta^{18} \mathrm{O}$ values increased again by $3 \%$ at the onset of GI 23 (cf. Capron et al., 2010). In the NALPS record this precursor event is centred at 104050 a (Fig. 3) and is characterized by a positive shift of $1.2 \%$ followed by a negative shift of $0.8 \%$ in $\delta^{18} \mathrm{O}$ and lasted for about $400 \mathrm{yr}(104170-103770 \mathrm{a})$. Going further back in the Last Glacial period, GI 24 shows two distinct negative (cooling) peaks of 100 to $200 \mathrm{yr}$ duration in NGRIP. Based on the GICC05modelext timescale the two minima occurred at $106770 \mathrm{a}$ and $106230 \mathrm{a}$, respectively. NALPS shows the first, more pronounced minimum in two stalagmites and the event is constrained to 107470 a based on the age model of stalagmite EXC3 (higher resolution and better chronology in this section than sample EXC4). The negative $\mathrm{O}$ isotope anomaly of $0.7 \%$ magnitude lasted for $380 \mathrm{yr}$ (107 620-
107240 a) and its progression is characterized by a rapid decrease and more gradual increase in both stalagmites. Capron et al. (2010) reported that no other interstadial was interrupted by comparable, short, cold events. In the NALPS record, however, two stalagmites document a distinct negative peak during GI 22 (see above). In this context the question whether GI 22 should be considered an interstadial or (only) a rebound-type structure following the long GI 23 (cf. Capron et al., 2010) can be raised again. At the end of GI 25 there is evidence of a rebound-type event in the NALPS record comparable to NGRIP (cf. Capron et al., 2010). The event lasted for ca. $180 \mathrm{yr}(111780-111600 \mathrm{a})$ and the maximum positive shift of $0.7 \%$ occurred at 111660 a based on NALPS (Fig. 3).

Regarding the secondary, sub-millennial events a relationship with the summer insolation at $65^{\circ} \mathrm{N}$ in connection with variable ice-sheet extensions was discussed (Capron et al., 2010). The rebound-type events are typically associated with relatively low summer insolation at the end of particularly long cooling phases during the GI progression. In contrast, the precursor-type events might be linked to insolation maxima; Capron et al. (2010) reported an in-phase relationship of the GI 21 precursor event with a relative maximum in $65^{\circ} \mathrm{N}$ summer insolation and a delay of ca. $2.5 \mathrm{kyr}$ of the GI 23 precursor relative to the preceding insolation maximum (after Laskar et al., 2004). Based on the NALPS chronology the precursor of GI 23 (maximum at $104050 \mathrm{a}$; cf. Fig. S2 in the Supplement) is delayed by ca. $1 \mathrm{kyr}$ when compared to the adjacent insolation maximum calculated by Laskar et al. (2004). The two precursor events of GI 21 recorded in NALPS (at 85360 and 84990 a; Fig. 3) preceded the insolation maximum by ca. 1 to $1.5 \mathrm{kyr}$ (Fig. S2). Using the NALPS chronology in relation to the summer insolation after Laskar et al. (2004) therefore suggests a shorter delay (ca. $1 \mathrm{kyr}$ ) of the GI 23 precursor event as compared to Capron et al. (2010). Our data support a preferential occurrence of the precursor events at times of maximum Northern Hemisphere summer insolation during the Last Glacial.

\section{Supplementary material related to this article is available online at: http://www.clim-past.net/7/1247/2011/ cp-7-1247-2011-supplement.zip.}

Acknowledgements. We are grateful to W. Breuss and A. Wolf who assisted in recovering stalagmite samples from the caves. M. Wimmer and A. Desch are acknowledged for laboratory assistance at the University of Innsbruck and B. Hardt, A. Burnett and M. Kelly for their help at the Minnesota Isotope Lab. A. Svensson and D. Fleitmann are acknowledged for their reviews and helpful comments. Funded by the Austrian Science Fund (FWF project P19788-N10 to CS).

Edited by: D.-D. Rousseau 


\section{References}

Ahn, J. and Brook, E. J.: Atmospheric $\mathrm{CO}_{2}$ and climate on millennial time scales during the last glacial period, Science, 322, 83-85, 2008.

Alley, R. B., Anandakrishnan, S., and Jung, P.: Stochastic resonance in the North Atlantic, Paleoceanography, 16, 190-198, 2001.

Arz, H. W., Lamy, F., Ganopolski, A., Nowaczyk, N., and Pätzold, J.: Dominant Northern Hemisphere climate control over millennial-scale glacial sea-level variability, Quaternary Sci. Rev., 26, 312-321, 2007.

Asmerom, Y., Polyak, V. J., and Burns, S. J.: Variable winter moisture in the southwestern United States linked to rapid glacial climate shifts, Nat. Geosci., 3, 114-117, 2010.

Auer, I., Böhm, R., Jurkovic, A., Lipa, W., Orlik, A., Potzmann, R., Schöner, W., Ungersböck, M., Matulla, C., Briffa, K., Jones, P., Efthymiadis, D., Brunetti, M., Nanni, T., Maugeri, M., Mercalli, L., Mestre, O., Moisselin, J.-M., Begert, M., MüllerWestermeier, G., Kveton, V., Bochnicek, O., Stastny, P., Lapin, M., Szalai, S., Szentimrey, T., Cegnar, T., Dolinar, M., GajicCapka, M., Zaninovic, K., Majstorovic, Z., and Nieplova, E.: HISTALP-historical instrumental climatological surface time series of the Greater Alpine Region, Int. J. Climatol., 27, 17-46, 2007.

Birchfield, G. E., Wang, H., and Rich, J. J.: Century/millennium internal climate variability: an ocean-atmosphere-continental ice sheet model, J. Geophys. Res., 99, 12459-12470, 1994.

Bond, G., Showers, W., Cheseby, M., Lotti, R., Almasi, P., deMenocal, P., Priore, P., Cullen, H., Hajdas, I., and Bonani, G.: A pervasive millennial-scale cycle in North Atlantic Holocene and Glacial climates, Science, 278, 1257-1266, 1997.

Braun, H., Christi, M., Rahmstorf, S., Ganopolski, A., Mangini, A., Kubatski, C., Roth, K., and Kromer, B.: Possible solar origin of the 1,470-year glacial climate cycle demonstrated in a coupled model, Nature, 438, 208-211, 2005.

Broecker, W. S., Bond, G., Klas, M., Bonani, G., and Wolfli, W.: A salt oscillator in the glacial Atlantic? The concept, Paleoceanography, 5, 469-477, 1990.

Capron, E., Landais, A., Chappellaz, J., Schilt, A., Buiron, D., Dahl-Jensen, D., Johnsen, S. J., Jouzel, J., Lemieux-Dudon, B., Loulergue, L., Leuenberger, M., Masson-Delmotte, V., Meyer, H., Oerter, H., and Stenni, B.: Millennial and sub-millennial scale climatic variations recorded in polar ice cores over the last glacial period, Clim. Past, 6, 345-365, doi:10.5194/cp-6-3452010, 2010.

Casty, C., Wanner, H., Luterbacher, J., Esper, J., and Böhm, R.: Temperature and precipitation variability in the European Alps since 1500, Int. J. Climatol., 25, 1855-1880, 2005.

Cheng, H., Edwards, R. L., Wang. X., Woodhead, J., Hellstrom, J., Wang, Y. J., and Kong, X. G.: A new generation of ${ }^{230}$ Th dating techniques: Tests of precision and accuracy, Goldschmidt Conference Abstracts, Geochim. Cosmochim. Ac., A157, 2008.

Cheng, H., Edwards, R. L., Broecker, W. S., Denton, G. H., Kong, X., Wang, Y., Zhang, R., and Wang, X.: Ice Age terminations, Science, 326, 248-252, 2009.

Clark, P. U., Marshall, S. J., Clarke, G. K. C., Hostetler, S. W., Licciardi, J. M., and Teller, J. T.: Freshwater forcing of abrupt climate change during the Last Glaciation, Science, 293, 283287,2001
Claussen, M., Ganopolski, A., Brovkin, V., Gerstengarbe, F.-W., and Werner, P.: Simulated global-scale response of the climate system to Dansgaard/Oeschger and Heinrich events, Clim. Dynam., 21, 361-370, 2003.

Clement, A. C. and Cane, M. A.: A role for the tropical Pacific coupled ocean-atmosphere system on Milankovitch and millennial timescales. Part I: A modelling study of tropical Pacific variability, in: Mechanisms of global climate change at millennial time scales, edited by: Geophys. Monogr. Ser., 112, edited by: P. U. Clark, Webb, R. S., and Keigwin, L. D., AGU, Washington D. C., 363-371, 1999.

Clement, A. C. and Peterson, L. C.: Mechanisms of abrupt climate change of the Last Glacial period, Rev. Geophys., 46, RG4002, doi:10.1029/2006RG000204, 2008.

Cruz, F. W., Karmann, I., Viana, O. Jr., Burns, S. J., Ferrari, J. A., Vuille, M., Sial, A. N., and Moreira, M. Z.: Stable isotope study of cave percolation waters in subtropical Brazil: Implications for paleoclimate inferences from speleothems, Chem. Geol., 220 245-262, 2005.

Dansgaard, W., Johnsen, S. J., Clausen, H. B., Dahl-Jensen, D., Gundestrup, N. S., Hammer, C. U., Hvidberg, C. S., Steffensen, J. P., Sveinbjörnsdottir, A. E., Jouzel, J., and Bond, G.: Evidence for general instability of past climate from a 250 -kyr ice-core record, Nature, 364, 218-220, 1993.

Ditlevsen, P. D. and Johnsen, S. J.: Tipping points: Early warning and wishful thinking, Geophys. Res. Lett., 37, L19703, doi:10.1029/2010GL044486, 2010.

Drysdale, R. N., Zanchetta, G., Hellstrom, J. C., Fallick, A. E., McDonald, J., and Cartwright, I.: Stalagmite evidence for the precise timing of North Atlantic cold events during the early last glacial, Geology, 35, 77-80, 2007.

Dykoski, C. A., Edwards, R. L., Cheng, H., Yuan, D., Cai, Y., Zhang, M., Lin, Y., Qing, J., An, Z., and Revenaugh, J.: A highresolution, absolute-dated Holocene and deglacial Asian monsoon record from Dongge Cave, China, Earth Planet. Sci. Lett., 233, 71-86, 2005.

Edwards, R. L., Chen, J. H., and Wasserburg, G. J.: ${ }^{238}{ }^{\mathrm{U}}{ }^{234} \mathrm{U}-$ ${ }^{230} \mathrm{Th}-{ }^{232} \mathrm{Th}$ systematics and the precise measurement of time over the past 500,000 years, Earth Planet. Sci. Lett., 81, 175$192,1986$.

Fleitmann, D., Cheng, H., Badertscher, S., Edwards, R. L., Mudelsee, M., Göktürk, O. M, Fankhauser, A., Pickering, R., Raible, C. C., Matter, A., Kramers, J., and Tüysüz, O.: Timing and climatic impact of Greenland interstadials recorded in stalagmites from northern Turkey, Geophys. Res. Lett., 36, L19707, doi:10.1029/2009GL040050, 2009.

Friedman, I. and O'Neil, J. R.: Compilation of stable isotope fractionation factors of geochemical interest, U.S. Geological Survey Professional Paper 440-KK, Data of Geochemistry, 6th Edn, 1977.

Ganopolski, A. and Rahmstorf, S.: Abrupt glacial climate changes due to stochastic resonance, Phys. Rev. Lett., 88, 038501-1038501-4, doi:10.1103/PhysRevLett.88.038501, 2002.

Genty, D., Blamart, D., Ouahdi, R., Gilmour, M., Baker, A., Jouzel, J., and Van-Exter, S.: Precise dating of Dansgaard-Oeschger climate oscillations in western Europe from stalagmite data, Nature, 421, 833-837, 2003. 
Grachev, A. M., Brook, E. J., and Severinghaus, J. P.: Abrupt changes in atmospheric methane at the MIS 5b-5a transition, Geophys. Res. Lett., 34, L20703, doi:10.1029/2007GL029799, 2007.

Grootes, P. M. and Stuiver, M.: Oxygen 18/16 variability in Greenland snow and ice with $10^{-3}$ - to $10^{5}$-year time resolution, J. Geophys. Res., 102, 26455-26470, 1997.

Grootes, P. M., Stuiver, M., White, J. W. C., Johnsen, S., and Jouzel, J.: Comparison of oxygen isotope records from the GISP2 and GRIP Greenland ice cores, Nature, 366, 552-554, 1993.

Huber, C., Leuenberger, M., Spahni, R., Flückiger, J., Schwander, J., Stocker, T.F., Johnsen, S., Landais, A., and Jouzel, J.: Isotope calibrated Greenland temperature record over Marine Isotope Stage 3 and ist relation to $\mathrm{CH}_{4}$, Earth Planet. Sci. Lett., 243, 504-519, 2006.

Humer, G., Rank, D., Trimborn, P., and Stichler, W.: Niederschlagsisotopenmessnetz Österreich, Monographien, Vol. 52, Umweltbundesamt, Vienna, 86 pp., 1995.

IAEA/WMO: Global Network of Isotopes in Precipitation, The GNIP database, accessible at: http://isohis.iaea.org, last access: 23 November, 2010.

Johnsen, S. J., Dahl-Jensen, D., Gundestrup, N., Steffensen, J. P., Clausen, H. B., Miller, H., Masson-Delmotte, V., Sveinbjörnsdottir, A. E., and White, J.: Oxygen isotope and palaeotemperature records from six Greenland ice-core stations: Camp Century, Dye-3, GRIP, GISP2, Renland and NorthGRIP, J. Quaternary Sci., 16, 299-307, 2001.

Kaiser, A., Scheifinger, H., Kralik, M., Papesch, W., Rank, D., and Stichler, W.: Links between meteorological conditions and spatial/temporal variations in long-term isotope records from the Austrian Precipitation Network, in: C\&SPaperSeries 13/P, International Conference "Study of Environmental Change using isotope techniques”, IAEA, Vienna, 23-27 April 2001, 67-77, 2001.

Kim, S. T. and O'Neil, J. R.: Equilibrium and nonequilibrium oxygen isotope effects in synthetic carbonates, Geochim. Cosmochim. Ac., 61, 3461-3475, 1997.

Lachniet, M. S.: Climatic and environmental controls on speleothem oxygen-isotope values, Quaternary Sci. Rev., 28, 412-432, 2009.

Lambeck, K. and Chappell, J.: Sea level change through the last glacial cycle, Science, 292, 679-686, 2001.

Lang, C., Leuenberger, M., Schwander, J., and Johnsen, S.: $16^{\circ} \mathrm{C}$ rapid temperature variation in Central Greenland 70,000 years ago, Science, 286, 934-937, 1999.

Laskar, J., Robutel, P., Joutel, F., Gastineau, M., Correia, A. C. M., and Levrard, B.: A long-term numerical solution for the insolation quantities of the Earth, Astron. Astrophys., 428, 261-285, 2004.

Loulergue, L., Schilt, A., Spahni, R., Masson-Delmotte, V., Blunier, T., Lemieux, B., Barnola, J.-M., Raynaud, D., Stocker, T. F., and Chappellaz, J.: Orbital and millennial-scale features of atmospheric $\mathrm{CH}_{4}$ over the past 800,000 years, Nature, 453, 383386, 2008

Lowe, J. J., Rasmussen, S. O., Björck, S., Hoek, W. Z., Steffensen, J. P., Walker, M. J. C., Yu, Z. C., and the INTIMATE group: Synchronisation of palaeoenvironmental events in the North Atlantic region during the Last Termination: a revised protocol recommended by the INTIMATE group, Quaternary Sci. Rev., 27,
6-17, 2008.

Meese, D. A., Gow, A. J., Alley, R. B., Grootes, P. M., Ram, M., Taylor, K. C., Zielinski, G. A., Bolzan, J. F., Mayewski, P. A., and Waddington, E. D.: The Greenland Ice Sheet Project 2 depth-age scale: Methods and results, J. Geophys. Res., 102, 26411-26423, 1997.

North Greenland Ice Core Project members: High-resolution record of Northern Hemisphere climate extending into the last interglacial period, Nature, 431, 147-151, 2004.

Paillard, D., Labeyrie, L., and Yiou, P.: Macintosh program performs time-series analysis, Eos Trans. AGU, 77, 379, doi:10.1029/96EO00259, 1996.

Peavoy, D. and Franzke, C.: Bayesian analysis of rapid climate change during the last glacial using Greenland ?180 data, Clim. Past, 6, 787-794, doi:10.5194/cp-6-787-2010, 2010.

$\mathrm{R}$ Development Core Team: The $\mathrm{R}$ Project for Statistical Computing, accessible at: http://www.r-project.org, last access: 23 November, 2010.

Rahmstorf, S.: Ocean circulation and climate during the past 120,000 years, Nature, 419, 207-214, 2002.

Rahmstorf, S.: Timing of abrupt climate change: a precise clock, Geophys. Res. Lett., 30, 1510, doi:10.1029/2003GL017115, 2003.

Richards, D. A. and Dorale, J. A.: Uranium series chronology and environmental applications of speleothems, in: Uranium series geochemistry, edited by: Bourdon, B., Henderson, G. M., Lundstrom, C. C., Turner, S., Rev. Mineral. Geochem., 52, 407-460, 2003.

Rousseau, D.-D., Kukla, J., and McManus, J.: What is what in the ice and the ocean?, Quaternary Sci. Rev., 25, 2025-2030, 2006.

Rozanski, K., Araguas-Araguas, L., and Gonfiantini, R.: Relation between long-term trends of oxygen-18 isotope composition of precipitation and climate, Science, 258, 981-985, 1992

Schmittner, A. and Galbraith, E. D.: Glacial greenhouse-gas fluctuations controlled by ocean circulation changes, Nature, 456 , 373-376, 2008.

Scholz, D. and Hoffmann, D.: StalAge - an algorithm designed for construction of speleothem age models, Quat. Geochronol., 6, 369-382, 2011.

Severinghaus, J. P., Sowers, T., Brook, E. J., Alley, R. B., and Bender, M. L.: Timing of abrupt climate change at the end of the Younger Dryas interval from thermally fractionated gases in Polar ice, Nature, 391, 141-146, 1998.

Shen, C.-C., Li, K.-S., Sieh, K., Natawidjaja, D., Cheng, H., Wang, X., Edwards, R. L., Lam, D. D., Hsieh, Y.-T., Fan, T.Y., Meltzner, A. J., Taylor, F. W., Quinn, T. M., Chiang, H.-W., and Kilbourne, K. H.: Variation of initial ${ }^{230} \mathrm{Th} /{ }^{232} \mathrm{Th}$ and limits of high precision U-Th dating of shallow-water corals, Geochim. Cosmochim. Ac., 72, 4201-4223, 2008.

Sodemann, H. and Zubler, E.: Seasonal and inter-annual variability of the moisture sources for Alpine precipitation during 19952002, Int. J. Climatol., 30, 947-961, 2010.

Spötl, C. and Mangini, A.: Stalagmite from the Austrian Alps reveals Dansgaard-Oeschger events during isotope stage 3: Implications for the absolute chronology of Greenland ice cores, Earth Planet. Sci. Lett., 203, 507-518, 2002.

Spötl, C. and Vennemann, T. W.: Continuous-flow isotope ratio mass spectrometric analysis of carbonate minerals, Rapid Commun. Mass Sp., 17, 1004-1006, 2003. 
Spötl, C., Mangini, A., and Richards, D. A.: Chronology and paleoenvironment of Marine Isotope Stage 3 from two high-elevation speleothems, Austrian Alps, Quaternary Sci. Rev., 25, $1127-$ 1136, 2006.

Spötl, C., Nicolussi, K., Patzelt, G., Boch, R., and DAPHNE Team: Humid climate during deposition of sapropel 1 in the Mediterranean Sea: Assessing the influence on the Alps, Global Planet. Change, 71, 242-248, 2010.

Steffensen, J. P., Andersen, K. K., Bigler, M., Clausen, H. B., DahlJensen, D., Fischer, H., Goto-Azuma, K., Hansson, M., Johnsen, S. J., Jouzel, J., Masson-Delmotte, V., Popp, V., Rasmussen, S. O., Röthlisberger, R., Ruth, U., Stauffer, B., Siggaard-Andersen, M.-L., Sveinbjörnsdóttir, A. E., Svensson, A., White, J. W. C.: High-resolution Greenland ice core data show abrupt climate change happens in few years, Science, 321, 680-684, 2008.

Svensson, A., Andersen, K. K., Bigler, M., Clausen, H. B., DahlJensen, D., Davies, S. M., Johnsen, S. J., Muscheler, R., Parrenin, F., Rasmussen, S. O., Rthlisberger, R., Seierstad, I., Steffensen, J. P., and Vinther, B. M.: A 60000 year Greenland stratigraphic ice core chronology, Clim. Past, 4, 47-57, doi:10.5194/cp-4-472008, 2008.

Von Grafenstein, U., Erlenkeuser, H., Brauer, A., Jouzel, J., and Johnsen, S. J.: A mid-European decadal isotope-climate record from 15,000 to 5000 years B. P., Science, 284, 1654-1657, 1999.
Wang, X., Auler, A. S., Edwards, R. L., Cheng, H., Ito, E., Wang, Y., Kong, X., and Solheid, M.: Millennial-scale precipitation changes in southern Brazil over the past 90,000 years, Geophys. Res. Lett., 34, L23701, doi:10.1029/2007GL031149, 2007.

Wang, Y. J., Cheng, H., Edwards, R. L., An, Z. S., Wu, J. Y., Shen, C.-C., and Dorale, J. A.: A high-resolution absolute dated late Pleistocene monsoon record from Hulu Cave, China, Science, 294, 2345-2348, 2001.

Wang, Y. J., Cheng, H., Edwards, R. L., Kong, X., Shao, X., Chen, S., Wu, J., Jiang, X., Wang, X., and An, Z.: Millennial- and orbital-scale changes in the East Asian monsoon over the past 224,000 years, Nature, 451, 1090-1093, 2008.

Wolff, E. W., Chappellaz, J., Blunier, T., Rasmussen, S. O., and Svensson, A.: Millennial-scale variability during the last glacial: The ice core record, Quaternary Sci. Rev., 29, 2828-2838, 2010.

Wunsch, C.: Abrupt climate change: An alternative view, Quaternary Res., 65, 191-203, 2006.

Xia, Z., Kong, X., Jiang, X., and Cheng, H.: Precise dating of East-Asian-Monsoon D/O events during 95-56 ka BP: Based on stalagmite data from Shanbao Cave at Shennongjia, China, Sci. China Ser. D, 50, 228-235, 2007. 